PUPT-1908

DAMTP-99-176

Imperial/TP/99-00/15

hep-th/0001222v2

January 2000

\title{
Conserved charges and supersymmetry in principal chiral and WZW models
}

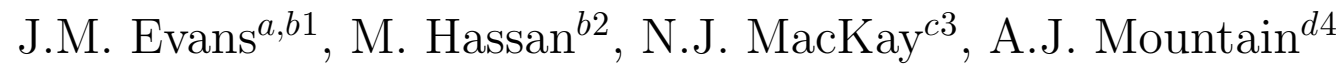 \\ a Joseph Henry Laboratories, Princeton University, Princeton NJ 08544, U.S.A. \\ ${ }^{b}$ DAMTP, University of Cambridge, Silver Street, Cambridge CB3 9EW, U.K. \\ c Department of Mathematics, University of York, York YO10 5DD, U.K. \\ ${ }^{d}$ Blackett Laboratory, Imperial College, Prince Consort Road, London SWY 2BZ, U.K.
}

\begin{abstract}
Conserved and commuting charges are investigated in both bosonic and supersymmetric classical chiral models, with and without Wess-Zumino terms. In the bosonic theories, there are conserved currents based on symmetric invariant tensors of the underlying algebra, and the construction of infinitely many commuting charges, with spins equal to the exponents of the algebra modulo its Coxeter number, can be carried out irrespective of the coefficient of the Wess-Zumino term. In the supersymmetric models, a different pattern of conserved quantities emerges, based on antisymmetric invariant tensors. The current algebra is much more complicated than in the bosonic case, and it is analysed in some detail. Two families of commuting charges can be constructed, each with finitely many members whose spins are exactly the exponents of the algebra (with no repetition modulo the Coxeter number). The conserved quantities in the bosonic and supersymmetric theories are only indirectly related, except for the special case of the WZW model and its supersymmetric extension.
\end{abstract}

\footnotetext{
${ }^{1}$ e-mail: evans@feynman.princeton.edu, J.M.Evans@damtp.cam.ac.uk

${ }^{2}$ e-mail: M.U.Hassan@damtp.cam.ac.uk

${ }^{3}$ e-mail:nm15@york.ac.uk

${ }^{4}$ e-mail: A.Mountain@ic.ac.uk
} 


\section{Introduction}

In previous work [1] we investigated local and non-local conserved charges in the bosonic principal chiral model (PCM) based on a compact classical Lie group. The non-local charges, forming the 'Yangian' quantum group, were shown to commute with the local charges. The algebra of the local charges themselves was found to be quite involved in general. Nevertheless, we were able to prove the existence of mutually commuting sets of local charges with spins equal to the exponents of the underlying classical Lie algebra modulo its Coxeter number. This is precisely the structure familiar from affine Toda field theory (see e.g. $[3,4]$ ) and to this extent the results provide a new perspective on properties [5] of the known S-matrices for PCMs [6].

In this paper we extend these earlier results in a number of directions. We begin by adding a Wess-Zumino (WZ) term to the bosonic PCM. A special case, at a critical value of the coupling, is the quantum-conformally-invariant WZW model. We show in section 2 how the results of [1] can be carried over in the presence of a WZ term with arbitrary coefficient. A more challenging problem is to find analogous results when fermions are added to the PCM (with or without a WZ term) so as to make the model supersymmetric, and it is to this that we devote the remainder of the paper. ${ }^{5}$ A number of novel features arise in conjunction with supersymmetry and a proper comparison with the bosonic theory is greatly facilitated by a thorough understanding of the special behaviour of the critical WZW and super WZW models, thus making contact with the results of section 2 .

The supersymmetric PCM is introduced in section 3 using both superspace and component fields. We then construct local and non-local conserved charges in the model, and explain the effects of adding a WZ term in these supersymmetric theories. In section 4 we investigate in detail the algebra of local currents, before proving the existence of sets of commuting charges whose spins are once again related to the exponents of the relevant Lie algebra. Various technical or supplementary results are collected in some appendices.

\footnotetext{
${ }^{5}$ Some preliminary results were described in conference talks [2].
} 


\section{The bosonic principal chiral and WZW models}

\subsection{The lagrangian, symmetries and currents}

The principal chiral model with a Wess-Zumino term can be defined by an action [7]

$$
\frac{\kappa}{2} \int d^{2} x \operatorname{Tr}\left(\partial_{\mu} g^{-1} \partial^{\mu} g\right)+\frac{\kappa}{3} \lambda \int_{B} \operatorname{Tr}\left(g^{-1} d g\right)^{3} .
$$

We shall refer to this theory as the principal-chiral-Wess-Zumino model or PCWZM. The field $g\left(x^{\mu}\right)$ is a function on spacetime (with coordinates $x^{\mu}$ ) taking values in some compact Lie group $\mathcal{G}$. This field must then be smoothly extended to a three-dimensional manifold $B$ whose boundary is spacetime in order to write the WZ term as above (where we have chosen to use differential form notation). The coupling constants $\lambda$ and $\kappa$ are dimensionless. Indeed, $\kappa$ is irrelevant classically, and may be assigned any numerical value without altering the theory. The combination $\kappa \lambda$ appearing in the coefficient of the WZ term must be

quantised in suitable units for a consistent quantum theory, but this will not be significant for us.

We shall consider only simple classical groups $\mathcal{G}=S U(N), S O(N), S p(N)(N$ even in the last case) with the field $g\left(x^{\mu}\right)$ a matrix in the defining representation. The corresponding Lie algebra $\mathbf{g}$ then consists of $N \times N$ complex matrices $X$ which obey

$$
\begin{array}{lll}
s u(N): & X^{\dagger}=-X, & \operatorname{Tr}(X)=0 \\
s o(N): & X^{*}=X, & X^{T}=-X \\
s p(N): & X^{\dagger}=-X, & X^{T}=-J X J^{-1}
\end{array}
$$

where $J$ is some chosen symplectic structure. In each case we introduce a basis of antihermitian generators $t^{a}$ for $\mathbf{g}$ with real structure constants $f^{a b c}$ and normalisations given by

$$
\left[t^{a}, t^{b}\right]=f^{a b c} t^{c}, \quad \operatorname{Tr}\left(t^{a} t^{b}\right)=-\delta^{a b}
$$

(Lie algebra indices will always be taken from the beginning of the alphabet.) For any $X \in \mathbf{g}$ we write

$$
X=t^{a} X^{a}, \quad X^{a}=-\operatorname{Tr}\left(t^{a} X\right)
$$

The advantage of writing the WZ term as a three-dimensional integral is that it makes manifest the continuous symmetry

$$
\mathcal{G}_{L} \times \mathcal{G}_{R}: \quad g \mapsto U_{\mathrm{L}} g U_{\mathrm{R}}^{-1}
$$


It is convenient to introduce the quantities

$$
E_{\mu}^{L}=\partial_{\mu} g g^{-1}, \quad E_{\mu}^{R}=-g^{-1} \partial_{\mu} g
$$

which take values in the Lie algebra $\mathbf{g}$ and transform only under the left and right factors of the symmetry group respectively. The equations of motion from the action (2.1) above state that the currents

$$
j_{\mu}^{L}=\kappa\left(E_{\mu}^{L}-\lambda \varepsilon_{\mu \nu} E^{L \nu}\right), \quad j_{\mu}^{R}=\kappa\left(E_{\mu}^{R}+\lambda \varepsilon_{\mu \nu} E^{R \nu}\right)
$$

are conserved:

$$
\partial^{\mu} j_{\mu}^{R}=\partial^{\mu} j_{\mu}^{L}=0 .
$$

We shall use both orthonormal coordinates $x^{0}=t$ and $x^{1}=x$ in two dimensions, with conventions $\eta^{00}=-\eta^{11}=1, \varepsilon^{01}=1$, and also light-cone coordinates and derivatives defined by

$$
x^{ \pm}=\frac{1}{2}(t \pm x), \quad \partial_{ \pm}=\partial_{t} \pm \partial_{x} .
$$

In terms of the latter, the definitions of the conserved currents become

$$
j_{ \pm}^{L}=\kappa(1 \mp \lambda) E_{ \pm}^{L}, \quad j_{ \pm}^{R}=\kappa(1 \pm \lambda) E_{ \pm}^{R}
$$

and the equations of motion are equivalent to either of the conditions

$$
\partial_{\mp} j_{ \pm}^{L}=\mp \frac{\kappa}{2}\left[j_{+}^{L}, j_{-}^{L}\right], \quad \partial_{\mp} j_{ \pm}^{R}=\mp \frac{\kappa}{2}\left[j_{+}^{R}, j_{-}^{R}\right] .
$$

The PCM is of course a special case of the PCWZM corresponding to the choice $\lambda=0$. WZW models are defined by the conditions $\lambda= \pm 1$, which we shall refer to as critical points, or critical values of the coupling (though we shall concentrate on the classical theories in this paper). The special nature of these critical points is evident in light-cone coordinates, since then $j_{ \pm}^{L}=j_{\mp}^{R}=0$ and the equations of motion become simply

$$
\partial_{\mp} j_{ \pm}^{R}=\partial_{ \pm} j_{\mp}^{L}=0
$$

We shall refer to conservation equations of this special type as holomorphic.

For our purposes it will be sufficient to deal with just one of the currents $j_{\mu}^{L}$ or $j_{\mu}^{R}$; we choose the right current and drop the label $R$ henceforth.

The classical action is conformally-invariant and as a result the energy-momentum tensor

$$
T_{\mu \nu}=-\frac{1}{2 \kappa}\left(\operatorname{Tr}\left(j_{\mu} j_{\nu}\right)-\frac{1}{2} \eta_{\mu \nu} \operatorname{Tr}\left(j_{\rho} j^{\rho}\right)\right)
$$


is not only conserved and symmetric but also traceless. In light-cone components it takes the familiar form

$$
T_{ \pm \pm}=-\frac{1}{2 \kappa} \operatorname{Tr}\left(j_{ \pm} j_{ \pm}\right), \quad T_{+-}=T_{-+}=0
$$

with

$$
\partial_{-} T_{++}=\partial_{+} T_{--}=0 .
$$

The WZ term does not contribute to the energy-momentum tensor because it is metricindependent.

Finally we remark on the discrete transformation

$$
\pi: g \mapsto g^{-1} \quad \Rightarrow \quad E_{\mu}^{L} \leftrightarrow E_{\mu}^{R},
$$

which exchanges $\mathcal{G}_{L}$ and $\mathcal{G}_{R}$ and which we shall consequently refer to as $\mathcal{G}$-parity. For the PCM, with $\lambda=0$, this is a symmetry of the lagrangian. For the more general case of the PCWZM it is not a symmetry by itself, but it is if combined with the usual spacetime parity transformation $x \mapsto-x$.

\subsection{Poisson brackets}

The canonical Poisson brackets of the conserved currents in the PCWZM are

$$
\begin{aligned}
& \left\{j_{0}^{a}(x), j_{0}^{b}(y)\right\}=f^{a b c} j_{0}^{c}(x) \delta(x-y)+2 \lambda \kappa \delta^{a b} \delta^{\prime}(x-y) \\
& \left\{j_{0}^{a}(x), j_{1}^{b}(y)\right\}=f^{a b c} j_{1}^{c}(x) \delta(x-y)+\left(1+\lambda^{2}\right) \kappa \delta^{a b} \delta^{\prime}(x-y) \\
& \left\{j_{1}^{a}(x), j_{1}^{b}(y)\right\}=f^{a b c}\left(2 \lambda j_{1}^{c}(x)-\lambda^{2} j_{0}^{c}(x)\right) \delta(x-y)+2 \lambda \kappa \delta^{a b} \delta^{\prime}(x-y)
\end{aligned}
$$

or, in light-cone coordinates,

$$
\begin{aligned}
\left\{j_{ \pm}^{a}(x), j_{ \pm}^{b}(y)\right\}= & \frac{1}{2} f^{a b c}(1 \pm \lambda)\left((3 \mp \lambda) j_{ \pm}^{c}(x)-(1 \pm \lambda) j_{\mp}^{c}(x)\right) \delta(x-y) \\
& \pm 2 \kappa(1 \pm \lambda)^{2} \delta^{a b} \delta^{\prime}(x-y) \\
\left\{j_{+}^{a}(x), j_{-}^{b}(y)\right\}= & \frac{1}{2} f^{a b c}\left((1-\lambda)^{2} j_{+}^{c}(x)+(1+\lambda)^{2} j_{-}^{c}(x)\right) \delta(x-y)
\end{aligned}
$$

We repeat that we are now dealing exclusively with the $R$ currents (the brackets of the $L$ currents with themselves are similar, while those of $L$ with $R$ are more complicated, but we shall need neither). These brackets may be derived in various ways; one method is sketched in an appendix, section 6.

Notice that different values of $\lambda$ result in genuinely different current algebras. In particular, we observe that at the critical values $\lambda= \pm 1$, with $j_{\mp}=0$, the surviving current 
component $j_{ \pm}$obeys a Kac-Moody algebra. The value of $\kappa$, on the other hand, is of no real significance. Corresponding to its appearance as an overall factor in the lagrangian, it could be eliminated from the current algebra by a simultaneous re-scaling of currents and Poisson brackets. We can take advantage of this when carrying out calculations, by setting $\kappa$ to some convenient numerical value.

\subsection{Local conserved charges and invariant tensors}

Consider the PCWZM based on $\mathcal{G}$ with arbitrary couplings $\kappa$ and $\lambda$. Let $d_{a_{1} a_{2} \ldots a_{m}}^{(m)}$ be any totally symmetric invariant tensor, (we shall not always indicate the rank $m$ explicitly) so that

$$
d_{c\left(a_{1} a_{2} \ldots a_{m-1}\right.} f_{\left.a_{m}\right) b c}=0 .
$$

For each such $d$-tensor there are holomorphic conservation equations

$$
\partial_{-}\left(d_{a_{1} a_{2} \ldots a_{m}} j_{+}^{a_{1}} j_{+}^{a_{2}} \ldots j_{+}^{a_{m}}\right)=\partial_{+}\left(d_{a_{1} a_{2} \ldots a_{m}} j_{-}^{a_{1}} j_{-}^{a_{2}} \ldots j_{-}^{a_{m}}\right)=0
$$

which follow immediately from (2.18) and from (2.10) written in the form

$$
\partial_{\mp} j_{ \pm}^{a}=\mp \frac{1}{2} \kappa f^{a b c} j_{+}^{b} j_{-}^{c}
$$

One special case is

$$
\partial_{-}\left(T_{++}^{n}\right)=\partial_{+}\left(T_{--}^{n}\right)=0
$$

which corresponds to even-rank invariant tensors constructed from Kronecker deltas:

$$
d_{a_{1} a_{2} \ldots a_{2 n-1} a_{2 n}}=\delta_{\left(a_{1} a_{2}\right.} \delta_{a_{3} a_{4}} \ldots \delta_{\left.a_{2 n-1} a_{2 n}\right)} .
$$

Such conservation laws hold in any classically conformally-invariant theory. More interesting are the equations

$$
\partial_{-} \operatorname{Tr}\left(j_{+}^{m}\right)=\partial_{+} \operatorname{Tr}\left(j_{-}^{m}\right)=0
$$

which correspond to choosing

$$
d_{a_{1} a_{2} \ldots a_{m}}=s_{a_{1} a_{2} \ldots a_{m}}:=\operatorname{s} \operatorname{Tr}\left(t^{a_{1}} t^{a_{2}} \ldots t^{a_{m}}\right) .
$$

with 'sTr' denoting the trace of a completely symmetrised product of matrices. For $s u(N)$ these tensors exist for any positive integer $m$. For $s o(N)$ or $s p(N)$ on the other hand, they are non-trivial only when $m$ is an even integer, vanishing identically when $m$ is odd. 
These observations lead us to a more detailed consideration of invariant tensors. There are infinitely many invariant tensors for each algebra $\mathbf{g}$, but only $\operatorname{rank}(\mathbf{g})$ independent or primitive $d$-tensors and Casimirs (see e.g. [9]), whose degrees equal the exponents of $\mathbf{g}$ plus one. For future reference we list the exponents of each classical algebra, together with the value of its Coxeter number, $h$ and the dimension of its fundamental representation, $N$ :

$$
\begin{array}{rcll}
a_{\ell}=s u(\ell+1) & 1,2,3, \ldots, \ell & h=\ell+1 & N=\ell+1 \\
b_{\ell}=s o(2 \ell+1) & 1,3,5, \ldots, 2 \ell-1 & h=2 \ell & N=2 \ell+1 \\
c_{\ell}=s p(2 \ell) & 1,3,5, \ldots, 2 \ell-1 & h=2 \ell & N=2 \ell \\
d_{\ell}=s o(2 \ell) & 1,3,5, \ldots, 2 \ell-3 ; \ell-1 & h=2 \ell-2 & N=2 \ell
\end{array}
$$

All other invariant tensors can be expressed as polynomials in the primitive tensors and the structure constants $f_{a b c}$. The choice of primitive tensors is certainly not unique. In particular, we can modify any given choice by adding terms involving compound tensors of the form $u_{\left(a_{1} \ldots a_{r}\right.} v_{\left.b_{1} \ldots b_{s}\right)}$. For the classical algebras, the primitive tensors can be chosen to be symmetrised traces $s_{a_{1} \ldots a_{m}}$ as in (2.23), with one exception. This exception is the Pfaffian invariant for $s o(2 \ell)$, which has rank $\ell$ and which can be written

$$
d_{a_{1} \ldots a_{\ell}}=p_{a_{1} \ldots a_{\ell}}:=\frac{1}{2^{\ell} \ell !} \epsilon_{i_{1} j_{1} \ldots i_{\ell} j_{\ell}}\left(t^{a_{1}}\right)_{i_{1} j_{1}} \ldots\left(t^{a_{\ell}}\right)_{i_{\ell} j_{\ell}} .
$$

Although the Pfaffian cannot itself be expressed as a polynomial in symmetrised traces, if $X$ is any element of the Lie algebra then it is always possible to express $\left(p_{a_{1} \ldots a_{\ell}} X^{a_{1}} \ldots X^{a_{\ell}}\right)^{2}=$ $\operatorname{det}(X)$ as a polynomial in traces of powers of $X$.

We are interested in the behaviour of the general conserved charges

$$
q_{ \pm s}=\int d_{a_{1} a_{2} \ldots a_{m}} j_{ \pm}^{a_{1}} j_{ \pm}^{a_{2}} \ldots j_{ \pm}^{a_{m}} d x
$$

which we label by their spins $\pm s= \pm(m-1)$, i.e. their eigenvalues under the Lorentz boost generator. In particular, the Poisson bracket algebra of these charges can be calculated directly from (2.17). This was done in [1] for the case $\lambda=0$ (the PCM) and it was observed that the ultralocal terms in (2.17) (those not involving $\delta^{\prime}$ ) never contribute, irrespective of their individual coefficients. Now the effect of introducing a WZ term is evidently just a modification of these coefficients by some $\lambda$-dependent functions. The arguments used in [1] therefore suffice to show that the ultralocal terms still do not contribute, even in the more general case $\lambda \neq 0$. An immediate consequence is that charges (2.26) with spins of opposite sign always commute, since the brackets of $j_{+}$with $j_{-}$involve only ultralocal terms. For charges whose spins have the same sign, the situation is more complicated, since there may then be a contribution from the non-ultralocal term (proportional to $\delta^{\prime}$ ) 
in (2.17). For example, $\left\{q_{s}, q_{r}\right\}$ is easily seen to be proportional to the integral of

$$
d_{a_{1} a_{2} \ldots a_{s} c}^{(s+1)} d_{b_{1} \ldots b_{r-1} e c}^{(r+1)} j_{+}^{a_{1}} j_{+}^{a_{2}} \ldots j_{+}^{a_{s}} j_{+}^{b_{1}} \ldots j_{+}^{b_{r-1}} \partial_{1} j^{e} .
$$

Let us focus for definiteness on charges of positive spin and introduce the notation

$$
\mathcal{J}_{m}=\operatorname{Tr}\left(j_{+}^{m}\right)=s_{a_{1} \ldots a_{m}} j_{+}^{a_{1}} \ldots j_{+}^{a_{m}}
$$

The Poisson brackets of these currents are readily calculated $[8,1]$, with the result

$$
\begin{aligned}
\left\{\mathcal{J}_{m}(x), \mathcal{J}_{n}(y)\right\}= & -2 m n \kappa(1+\lambda)^{2}\left[\left(\mathcal{J}_{m+n-2}(x)-\frac{1}{N} \mathcal{J}_{m-1}(x) \mathcal{J}_{n-1}(x)\right) \delta^{\prime}(x-y)\right. \\
& \left.+\frac{n-1}{n+m-2} \mathcal{J}_{m+n-2}^{\prime}(x) \delta(x-y)-\frac{1}{N} \mathcal{J}_{m-1}(x) \mathcal{J}_{n-1}^{\prime}(x) \delta(x-y)\right]
\end{aligned}
$$

This holds for each of the algebras $s u(N), s o(N)$ and $s p(N)$, though in the latter two cases the integers $m$ and $n$ must be taken to be even and the terms with coefficients $1 / N$ then vanish. By virtue of our earlier remarks concerning primitive invariant tensors, any positive-spin current of the general type (2.19) can be expressed as a polynomial in a finite number of the currents $\mathcal{J}_{m}$, together with, for the case of $s o(2 \ell)$, the Pfaffian current, which we write

$$
\mathcal{P}_{\ell}=p_{a_{1} \ldots a_{\ell}} j_{+}^{a_{1}} \ldots j_{+}^{a_{\ell}}
$$

We remarked earlier that the Pfaffian can also be expressed in terms of symmetrised traces, but only by taking a square root of a polynomial. In practice such an expression may be rather inconvenient, although for our purposes this will be sufficient. Direct ways of calculating Poisson brackets involving the Pfaffian current are described in [1].

Our aim now is to identify certain natural families of local conserved charges of type (2.26) which all have vanishing Poisson brackets with one another. For the orthogonal and symplectic algebras, for which the $1 / N$ terms in (2.29) vanish, we see that the currents $\mathcal{J}_{m}$ already yield commuting charges. This leaves out the case of $s u(N)$, however, and also the Pfaffian primitive invariant in $s o(2 \ell)$. In the next section we shall give a universal formula which defines sets of commuting charges in any PCWZM based on a classical algebra.

To conclude this section, a number of comments are in order. Firstly, we should mention in passing the existence of a much larger set of conserved local quantities in any PCWZM, consisting of differential polynomials in the currents (2.19). These will have no role to play in the remainder of this paper. More importantly for us, there is a quite different but no less dramatic increase in the number of local charges for the special case of the 
WZW model. With $\lambda=+1$, say, the Lie-algebra-valued current $j_{+}^{a}$ is itself holomorphic, as in (2.11). Consequently, the tensor $d$ appearing in (2.19) need no longer be invariant in order to give rise to a holomorphic current: arbitrary polynomials in the components $j_{+}^{a}$ are automatically holomorphic. We shall not investigate this larger set of currents in the WZW model in any detail, but knowledge of its existence will prove helpful later.

Finally, there is a related point concerning the quantum theory. Away from the critical points $\lambda= \pm 1$, the scale-invariance of the classical PCWZM will be broken quantummechanically [7]. We would therefore expect that the quantum versions of the classical conservation laws (2.19) are no longer of holomorphic form, but rather modified by anomalies (see e.g. [22]). Our detailed knowledge of the quantum conservation laws is rather incomplete - see [1] for a summary of the PCM. At critical values of the coupling $\lambda= \pm 1$, however, we obtain the quantum conformally-invariant WZW theories, and we then expect the holomorphic form of the conservation equations to persist quantum-mechanically.

\subsection{Commuting families of local charges}

We now explain how the main results of [1] regarding commuting sets of local charges can be generalised to the PCWZMs.

For each of the classical algebras $a_{\ell}, b_{\ell}, c_{\ell}$ and $d_{\ell}$, we introduce the generating functions $A(x, \mu)$ and $F(x, \mu)$ by

$$
A(x, \mu)=\exp F(x, \mu)=\operatorname{det}\left(1-\mu j_{+}(x)\right)
$$

so that

$$
F(x, \mu)=\operatorname{Tr} \log \left(1-\mu j_{+}(x)\right)=-\sum_{r=2}^{\infty} \frac{\mu^{r}}{r} \mathcal{J}_{r}(x) .
$$

Now define polynomials $\mathcal{K}_{s+1}$ in the currents $\mathcal{J}_{m}$, which have homogeneous spin $s+1$, by

$$
-\frac{1}{s+1} \mathcal{K}_{s+1}=\left.A(x, \mu)^{s / h}\right|_{\mu^{s+1}}=\left.\exp \frac{s}{h} F(x, \mu)\right|_{\mu^{s+1}}
$$

where $h$ is the Coxeter number of the algebra, as given in $(2.24)$. Note that $A(x, \mu)$ is a polynomial in $\mu$ with degree equal to the dimension of the defining representation, whereas $F(x, \mu)$ and fractional powers of $A(x, \mu)$ must be defined as power series in $\mu$ with infinitely many terms. In defining $\mathcal{K}_{s+1}$ by extracting the indicated coefficient, the generating function is to be expanded in ascending powers of $\mu$ :

$$
A(x, \mu)^{s / h}=\sum_{r=0}^{\infty} \mu^{r} \mathcal{A}_{r}^{(s / h)}(x) \quad \Rightarrow \quad-\frac{1}{s+1} \mathcal{K}_{s+1}=\mathcal{A}_{s+1}^{(s / h)}
$$


The coefficient of the current in $(2.33)$ is $\operatorname{chosen}^{6}$ to ensure that $\mathcal{K}_{n}$ has leading term $\mathcal{J}_{n}$. The first few examples of these new currents are:

$$
\begin{aligned}
\mathcal{K}_{2} & =\mathcal{J}_{2} \\
\mathcal{K}_{3} & =\mathcal{J}_{3} \\
\mathcal{K}_{4} & =\mathcal{J}_{4}-\frac{3}{2 h} \mathcal{J}_{2}^{2} \\
\mathcal{K}_{5} & =\mathcal{J}_{5}-\frac{10}{3 h} \mathcal{J}_{3} \mathcal{J}_{2} \\
\mathcal{K}_{6} & =\mathcal{J}_{6}-\frac{5}{3 h} \mathcal{J}_{3}^{2}-\frac{15}{4 h} \mathcal{J}_{4} \mathcal{J}_{2}+\frac{25}{8 h^{2}} \mathcal{J}_{2}^{3}
\end{aligned}
$$

These formulas apply to all algebras, though for the orthogonal and symplectic cases we must keep in mind that only the currents of even spin are non-vanishing.

The new currents $\mathcal{K}_{s+1}$ are non-trivial precisely when $s$ is an exponent of the algebra $\mathbf{g}$ modulo its Coxeter number $h$. If we consider $\mathbf{g}=s u(N)$, the currents vanish when $s / h$ is an integer, since $A(x, \mu)^{s / h}$ is then a polynomial in $\mu$ of degree $s$ (rather than a power series in $\mu$ ) and so the definition (2.33) becomes empty. For the orthogonal and symplectic algebras, $h$ is always even, whereas the currents are non-vanishing only when $s$ is odd. We have thus defined infinite sequences of currents or charges, each associated to a primitive invariant tensor of type (2.23) of $\mathbf{g}$ and with spins repeating $\bmod h$ within each sequence.

The only primitive invariant missing from the discussion is the Pfaffian for $\mathbf{g}=s o(2 \ell)$. To include this on the same footing, we must find an infinite family of currents $\mathcal{P}_{\ell+a h}$ where $a=0,1,2, \ldots$ and $h=2(\ell-1)$ is the Coxeter number of $s o(2 \ell)$. It is not immediately obvious how this should be done, but it turns out that this final sequence is already contained in the formulas given above, in the following rather surprising way.

First note that when $\mathbf{g}=s o(2 \ell)$ the term of highest degree in $A(x, \mu)$ is $\mu^{2 \ell} \mathcal{P}_{\ell}^{2}$. On extracting this factor from $A(x, \mu)$, we are left with a polynomial in $1 / \mu$, and this allows us to consider an expansion for $A(x, \mu)^{s / h}$ in decreasing, rather than increasing, powers of $\mu$. Moreover, our earlier formula, on the right-hand-side of (2.33), still makes perfect sense with $h=2(\ell-1)$ if we take $s=(2 a+1)(\ell-1)$ with $a$ a non-negative integer, and we can use it to define

$$
\mathcal{P}_{s+1}=\left.A(x, \mu)^{s / h}\right|_{\mu^{s+1}}
$$

\footnotetext{
${ }^{6}$ In [1] we adopted a number of different normalizations for these currents, as well as for the Pfaffian in (2.25) and (2.30). Such factors are obviously irrelevant to whether the corresponding charges commute, but they must be borne in mind when making comparisons with certain formulas in [1].
} 
To be more explicit, the expansion in inverse powers of $\mu$ takes the form

$$
A(x, \mu)^{s / h}=\mu^{(2 a+1) \ell} \sum_{r=0}^{\infty} \mu^{-r} \mathcal{A}_{-r}^{(s / h)}(x) \quad \Rightarrow \quad \mathcal{P}_{\ell+a h}=\mathcal{A}_{-2 a}^{(s / h)}
$$

for $a=0,1,2, \ldots$. The first of these $(a=0)$ is indeed the Pfaffian, and the following members of the sequence are its desired generalisations.

The importance of the new currents defined in (2.33) and (2.36) is that their charges $\int \mathcal{K}_{s+1} d x$ and $\int \mathcal{P}_{s+1} d x$ all have vanishing Poisson brackets in the PCWZM. This was proved for the case $\lambda=0$ in [1] by calculating the Poisson brackets of $A(x, \mu)^{p}$ and $A(y, \nu)^{q}$ and then showing that certain coefficients in the expansions were zero provided the powers $p$ and $q$ were chosen appropriately. The extension from the PCM to the PCWZM is straightforward once we know the effect of the WZ term, as given by the dependence on $\lambda$, in the brackets (2.17) and (2.29). Indeed, since $\lambda$ appears in (2.29) only through the overall factor $(1+\lambda)^{2}$, it will appear in just the same way in brackets of $F, A$, powers of $A$, and hence in the brackets of the conserved charges. Since changing zero by an overall factor still gives zero, the calculations of [1] imply that the charges commute in the PCWZM as well as in the PCM.

There are actually more general sets of commuting charges for the algebras $s o(2 \ell+1)$ and $s p(2 \ell)$, which correspond to replacing $1 / h$ by an arbitrary real number $\alpha$ in $(2.33)$. The same freedom exists for the trace-type currents in the case of $s o(2 \ell)$, as far as their mutual commutation is concerned, but requiring them to commute with the Pfaffian charges fixes $\alpha=1 / h$.

The formula for the currents $\mathcal{K}_{m}$ in terms of $\mathcal{J}_{m}$ amounts to a new choice $d_{a_{1} \ldots a_{m}}=k_{a_{1} \ldots a_{m}}$ in (2.19); thus

$$
\mathcal{K}_{m}=k_{a_{1} \ldots a_{m}} j_{+}^{a_{1}} \ldots j_{+}^{a_{m}} .
$$

Moreover the expressions for these new tensors in terms of symmetric traces, of the form,

$$
k_{a_{1} \ldots a_{m}}=s_{a_{1} \ldots a_{m}}+(\text { compound terms })
$$

may be regarded as a new choice for the primitive symmetric invariants. In addition to $k_{a_{1} a_{2}}=s_{a_{1} a_{2}}$ and $k_{a_{1} a_{2} a_{3}}=s_{a_{1} a_{2} a_{3}}$ we have the first few non-trivial examples

$$
\begin{aligned}
k_{a_{1} a_{2} a_{3} a_{4}} & =s_{a_{1} a_{2} a_{3} a_{4}}-\frac{3}{2 h} s_{\left(a_{1} a_{2}\right.} s_{\left.a_{3} a_{4}\right)} \\
k_{a_{1} a_{2} a_{3} a_{4} a_{5}} & =s_{a_{1} a_{2} a_{3} a_{4} a_{5}}-\frac{10}{3 h} s_{\left(a_{1} a_{2} a_{3}\right.} s_{\left.a_{4} a_{5}\right)}
\end{aligned}
$$




$$
\begin{aligned}
k_{a_{1} a_{2} a_{3} a_{4} a_{5} a_{6}=}= & s_{a_{1} a_{2} a_{3} a_{4} a_{5} a_{6}}-\frac{5}{3 h} s_{\left(a_{1} a_{2} a_{3}\right.} s_{\left.a_{4} a_{5} a_{6}\right)} \\
& -\frac{15}{4 h} s_{\left(a_{1} a_{2} a_{3} a_{4}\right.} s_{\left.a_{5} a_{6}\right)}+\frac{25}{8 h^{2}} s_{\left(a_{1} a_{2}\right.} s_{a_{3} a_{4}} s_{\left.a_{5} a_{6}\right)}
\end{aligned}
$$

The vanishing of the charge Poisson brackets implies the algebraic property

$$
k_{\left(a_{1} \ldots a_{s}\right.}^{(s+1)}{ }^{c} k_{\left.a_{s+1} \ldots a_{s+r-1}\right) b c}^{(r+1)}=k_{\left(a_{1} \ldots a_{s}\right.}^{(s+1)}{ }^{c} k_{\left.a_{s+1} \ldots a_{s+r-1} b\right) c}^{(r+1)},
$$

since this is a necessary and sufficient condition for the integrand in (2.27) to be a total derivative. This observation will prove useful later.

\subsection{Non-local charges}

In this section we construct the non-local charges in the PCWZM and then show that they commute with any charge arising from a local current (2.19).

Recall that the PCM [1] contains infinitely many conserved, non-local charges, which generate a Yangian $Y(\mathbf{g})$ [11]. In fact there are two copies of this structure, constructed either from $j_{\mu}^{L}$ or $j_{\mu}^{R}$, and so the model has a charge algebra $Y_{L}(\mathbf{g}) \times Y_{R}(\mathbf{g})$. The charges are constructed [12] (again for either $L$ or $R$; we specialise to $R$ ) from an infinite sequence of currents $j_{\mu}^{(r)}$, with $r=0,1,2, \ldots$, obeying

$$
\partial^{\mu} j_{\mu}^{(r)}=0 \quad \Leftrightarrow \quad j_{ \pm}^{(r)}= \pm \partial_{ \pm} \chi^{(r)}
$$

for some scalar functions $\chi^{(r)}$. They are defined by

$$
j_{ \pm}^{(0)}=j_{ \pm}, \quad j_{ \pm}^{(1)}=(1 \pm \lambda)\left(\partial_{ \pm} \chi^{(0)}-\frac{1}{2}\left[E_{ \pm}, \chi^{(0)}\right]\right)
$$

and

$$
j_{\mu}^{(r+1)}=\nabla_{\mu} \chi^{(r)}=(1 \pm \lambda)\left(\partial_{ \pm} \chi^{(r)}-\left[E_{ \pm}, \chi^{(r)}\right]\right), \quad r \geq 1,
$$

which also defines the covariant derivative $\nabla_{\mu}$. Note the factor of one half in the definition of $j_{\mu}^{(1)}$ which is needed to ensure that it is conserved. Conservation of $j_{\mu}^{(r)}$ for $r>1$ is easily established by induction, using the properties $\left[\partial_{\mu}, \nabla^{\mu}\right]=\left[\nabla_{\mu}, \nabla_{\nu}\right]=0$. The first two conserved charges are $^{7}$

$$
\begin{aligned}
Q^{(0) a} & =\int d x j_{0}^{a}(x) \\
Q^{(1) a} & =\int d x\left(j_{1}^{a}(x)+\lambda j_{0}^{a}(x)-\frac{1}{2 \kappa} f^{a b c} \int^{x} d y j_{0}^{b}(x) j_{0}^{c}(y)\right) .
\end{aligned}
$$

\footnotetext{
7 Bernard [13] writes down a closely related procedure in which, using the freedom inherent in the Yangian, he effectively subtracts $2 \lambda Q^{(0) a}$ from $Q^{(1) a}$, so that in the conformal limit $Q^{(1) a}$ is purely nonlocal. See also [16, 17].
} 
We would expect $Q^{(0)}$ and $Q^{(1)}$ to form a Yangian, as in the $\lambda=0$ case, and the ultralocal parts of the Poisson brackets have the behaviour necessary for this. However, there is a problem with the non-ultralocal terms (the derivatives of delta functions). When $\lambda=0$ the only non-ultralocal term appears in the $\left\{j_{0}, j_{1}\right\}$ bracket. Ambiguities in the charge brackets can then be resolved by letting each charge be defined with a range of integration from $-L$ to $L$, then letting $L \rightarrow \infty$. The brackets which define the Yangian are then independent of the order in which the limits $L_{1} \rightarrow \infty, L_{2} \rightarrow \infty$ for the two charges are taken. For $\lambda \neq 0$ this is no longer possible. There are non-ultralocal terms in each of the current brackets and these lead to an ambiguity in $\left\{Q^{(0) a}, Q^{(1) b}\right\}$ which cannot be resolved, so that the Yangian is no longer well-defined - an issue which remains to be understood.

Although the algebraic structure generated by $Q^{(0) b}$ and $Q^{(1) b}$ may be ambiguous when $\lambda \neq 0$, their brackets with a general local charge $q_{s}$ given by $(2.26)$ are still well-defined. It is straightforward to show that $\left\{q_{s}, Q^{(0) b}\right\}=0$. In considering $\left\{q_{s}, Q^{(1) b}\right\}$, recall [1] that in the $\lambda=0$ case the local and non-local contributions from $Q^{(1) b}$ conspired to cancel. The same now occurs with $\lambda \neq 0$ : the $\int j_{1}^{(0) b}$ terms yield extra contributions

$$
\int d x f^{a_{1} b c}\left(2 \lambda j_{1}^{(0) c}-\lambda^{2} j_{0}^{(0) c}\right) d_{a_{1} a_{2} \ldots a_{s+1}} j_{+}^{(0) a_{2}} j_{+}^{(0) a_{3}} \ldots j_{+}^{(0) a_{s+1}}
$$

the $\lambda j_{0}^{(0) b}$ terms give nothing new, and the non-local terms give extra contributions

$$
\int d x f^{a_{1} b c}\left(2 \lambda j_{0}^{(0) c}+\lambda^{2} j_{0}^{(0) c}\right) d_{a_{1} a_{2} \ldots a_{s+1}} j_{+}^{(0) a_{2}} j_{+}^{(0) a_{3}} \ldots j_{+}^{(0) a_{s+1}}
$$

(we are assuming $s>0$ ). The $\lambda^{2}$ terms cancel when these expressions are added, and the others sum to produce a factor $2 \lambda j_{+}^{(0) c}$, which gives zero on using invariance of $d$.

\section{The super PCM (and super WZW model)}

In the rest of this paper we shall consider supersymmetric extensions of the bosonic models discussed above. Supersymmetric principal chiral models (SPCMs) have long been known to be integrable (see e.g. [18]) but our understanding of them is much less complete than for their bosonic counterparts. Indeed, an S-matrix for the $S U(N)$ SPCM has been conjectured (and tested) only fairly recently [19], and it is far from clear how this particular construction could be generalised to other groups. It is interesting therefore to consider whether a study of local charges, and the possible emergence of some version of Dorey's rule $[3,4]$, might be helpful for the eventual determination of these exact scattering theories. 
The super WZW model by contrast has received considerable attention in the context of conformal field theory-see e.g. [20, 21].

In the last section we showed that the addition of a WZ term to the bosonic PCM ultimately has no effect on the existence of the conserved currents (2.19) nor on the construction of commuting local charges based on the currents (2.33). Moreover, we found that the model displayed qualitatively different behaviour only at the critical WZW point. A similar picture emerges for the supersymmetric extensions of these models. Because the formulas and calculations are considerably more complicated for the supersymmetric theories, we shall simplify the presentation by concentrating for the most part on the SPCM, without a WZ term. Where the presence of a WZ term becomes significant, however, we shall mention its effects explicitly. In particular, some special features of the super WZW theory are important in clarifying the relationship between the bosonic and supersymmetric cases.

\subsection{Superspace and conserved currents}

To write down the SPCM in a manifestly supersymmetric way we shall use superspace, with coordinates $\left(x^{\mu}, \theta^{+}, \theta^{-}\right)$. The additional fermionic coordinates $\theta^{ \pm}$are real Grassmann numbers, with supercharges $Q_{ \pm}=\partial_{\theta^{ \pm}}+i \theta^{ \pm} \partial_{ \pm}$and supercovariant derivatives $D_{ \pm}=$ $\partial_{\theta^{ \pm}}-i \theta^{ \pm} \partial_{ \pm}$. Each index \pm signifies one unit of Lorentz spin on a bosonic object, but a 1/2-unit of spin on a fermionic object. Upper and lower indices denote opposite Lorentz weights. (A fuller discussion is given in an appendix, section 7.)

In analysing the SPCM we will need to understand the implications of conservation equations in superspace, which have the general form

$$
D_{+} J_{-}-D_{-} J_{+}=0
$$

Let us take the current components $J_{ \pm}$to be fermionic superfields, each carrying a single Lorentz spinor index, with all other possible internal or Lorentz indices suppressed (more elaborate possibilities can be dealt with straightforwardly). To examine the $x$-space content of the above equation we define the component expansions

$$
\begin{aligned}
& J_{+}=\alpha_{+}+\theta^{+} j_{+}+\theta^{-} u+i \theta^{+} \theta^{-} \beta_{+}, \\
& J_{-}=\alpha_{-}+\theta^{-} j_{-}+\theta^{+} v+i \theta^{-} \theta^{+} \beta_{-}
\end{aligned}
$$


in which all the fields are real, with $\alpha_{ \pm}$and $\beta_{ \pm}$fermionic, while $j_{ \pm}, u, v$ are bosonic. Now (3.1) is equivalent to

$$
\begin{aligned}
\partial_{+} j_{-}+\partial_{-} j_{+} & =0 \\
\partial_{-} \alpha_{+} & =\beta_{-} \\
\partial_{+} \alpha_{-} & =\beta_{+} \\
u & =v .
\end{aligned}
$$

The first of these equations is the usual conservation equation for a bosonic current with light-cone components $j_{ \pm}$, and the corresponding conserved charge can be written either as an integral in $x$-space or directly in superspace:

$$
B=\int\left(d x^{+} j_{+}-d x^{-} j_{-}\right)=\int\left(d x^{+} d \theta^{+} J_{+}-d x^{-} d \theta^{-} J_{-}\right)
$$

where the $x$-integrals are understood to be taken over a space-like curve.

The remaining equations (3.4)-(3.6) do not, in general, express any additional conservation laws. This is consistent with the fact that the charge $B$ is always invariant under supersymmetry: $\delta_{\epsilon} B=0$. This follows from either of the expressions in (3.7), indeed, it follows from the second expression simply because $J_{ \pm}$are superfields. Thus it is not possible to discover a 'superpartner charge' to $B$ in this manner. (See also [14].)

Nevertheless, there are some important special circumstances in which we know something more about the superspace current, thereby giving extra content to (3.4)-(3.6) and implying that there are additional conserved charges which are superpartners to $B$. The simplest example is that of a holomorphic conservation law in superspace, for which $J_{-}=0$. In this case we clearly have

$$
D_{-} J_{+}=0, \quad \Rightarrow \quad J_{+}=\alpha_{+}+\theta^{+} j_{+} \quad \text { with } \quad \partial_{-} j_{+}=\partial_{-} \alpha_{+}=0 .
$$

Now in addition to the previous bosonic charge $B$ we also have a fermionic charge:

$$
F=\int d x^{+} \alpha_{+}, \quad B=\int d x^{+} j_{+} .
$$

The action of supersymmetry on the currents is

$$
\delta_{\epsilon} \alpha_{+}=\epsilon^{+} j_{+}, \quad \delta_{\epsilon} j_{+}=\epsilon^{+} \partial_{+} \alpha_{+}
$$

and so on the corresponding charges we have

$$
\delta_{\epsilon} F=\epsilon^{+} B, \quad \delta_{\epsilon} B=0 .
$$


Notice that our earlier conclusion regarding invariance of $B$ is unaltered.

These observations will be useful shortly. Some more extensive comments about superspace conservation laws are collected in an appendix, section 7.

\subsection{The SPCM lagrangian and symmetries}

To define the supersymmetric principal chiral model (SPCM) we introduce a superfield $G(x, \theta)$ with values in $\mathcal{G}$. The superspace lagrangian is

$$
\mathcal{L}=\frac{1}{2} \operatorname{Tr}\left(D_{+} G^{-1} D_{-} G\right)
$$

where we immediately set the overall coupling constant factor to unity. This has a continuous symmetry

$$
\mathcal{G}_{L} \times \mathcal{G}_{R}: \quad G \mapsto U_{L} G U_{R}^{-1}
$$

and there are conserved, Lie algebra-valued superspace currents associated with each factor. As with the bosonic PCM, it will suffice to deal with just one of these, which we choose to be the current corresponding to $\mathcal{G}_{R}$, namely,

$$
J_{ \pm}=-i G^{-1} D_{ \pm} G
$$

(the current for $\mathcal{G}_{L}$ is then $-G J_{ \pm} G^{-1}$ ). In addition to the superspace conservation equation (3.1) we have identically a zero-curvature condition in superspace:

$$
D_{+} J_{-}+D_{-} J_{+}+i\left\{J_{+}, J_{-}\right\}=0 \text {. }
$$

Combining these, the superspace equations of motion of the SPCM can be written

$$
D_{+} J_{-}=D_{-} J_{+}=-\frac{i}{2}\left\{J_{+}, J_{-}\right\}
$$

To reveal the component ( $x$-space) content of the super PCM we can expand

$$
G(x, \theta)=g(x)\left(1+i \theta^{+} \psi_{+}(x)+i \theta^{-} \psi_{-}(x)+i \theta^{+} \theta^{-} \sigma(x)\right) .
$$

The fermions $\psi_{ \pm}(x)$ take values in $\mathbf{g}$ and are the superpartners of the group-valued fields $g(x)$. The $\mathcal{G}_{L} \times \mathcal{G}_{R}$ symmetry acts on the component fields by $g \mapsto U_{L} g U_{R}^{-1}$, and $\psi_{ \pm} \mapsto$ $U_{R} \psi_{ \pm} U_{R}^{-1}$, so that the fermions transform only under the $\mathcal{G}_{R}$ factor of the symmetry group. ${ }^{8}$

\footnotetext{
${ }^{8}$ An alternative component expansion $G=\exp \left(i \theta^{+} \tilde{\psi}_{+}+i \theta^{-} \tilde{\psi}_{-}+i \theta^{+} \theta^{-} \tilde{\sigma}\right) g$ would result in $g \mapsto U_{L} g U_{R}^{-1}$ and $\tilde{\psi}_{ \pm} \mapsto U_{L} \tilde{\psi}_{ \pm} U_{L}^{-1}$. The choices of fermions are of course related by $\psi_{ \pm}=g^{-1} \tilde{\psi}_{ \pm} g$
} 
The field $\sigma(x)$ turns out to be auxiliary, with an algebraic field equation. After its elimination, the final form of the component lagrangian is

$$
\begin{aligned}
L= & -\frac{1}{2} \operatorname{Tr}\left(g^{-1} \partial_{+} g g^{-1} \partial_{-} g+i \psi_{+} \partial_{-} \psi_{+}+i \psi_{-} \partial_{+} \psi_{-}\right. \\
& \left.+\frac{1}{2} i \psi_{+}\left[g^{-1} \partial_{-} g, \psi_{+}\right]+\frac{1}{2} i \psi_{-}\left[g^{-1} \partial_{+} g, \psi_{-}\right]+\frac{1}{2} \psi_{+}^{2} \psi_{-}^{2}\right) .
\end{aligned}
$$

The component equations of motion which follow from this can equally-well be read off from the current conservation equations. We first calculate

$$
\begin{aligned}
J_{+}=-i G^{-1} D_{+} G= & \psi_{+}-\theta^{+}\left(g^{-1} \partial_{+} g+i \psi_{+}^{2}\right)-\frac{1}{2} i \theta^{-}\left\{\psi_{+}, \psi_{-}\right\} \\
& -i \theta^{+} \theta^{-}\left(\partial_{+} \psi_{-}+\left[g^{-1} \partial_{+} g, \psi_{-}\right]+\frac{1}{2} i\left[\psi_{+}^{2}, \psi_{-}\right]\right) \\
J_{-}=-i G^{-1} D_{-} G= & \psi_{-}-\theta^{-}\left(g^{-1} \partial_{-} g+i \psi_{-}^{2}\right)-\frac{1}{2} i \theta^{+}\left\{\psi_{+}, \psi_{-}\right\} \\
& -i \theta^{-} \theta^{+}\left(\partial_{-} \psi_{+}+\left[g^{-1} \partial_{-} g, \psi_{+}\right]+\frac{1}{2} i\left[\psi_{-}^{2}, \psi_{+}\right]\right)
\end{aligned}
$$

(having already eliminated the auxiliary field). We can then compare these component expansions to (3.2), and we find that the equations (3.3-3.6) imply that the bosonic current

$$
j_{ \pm}=-\left(g^{-1} \partial_{ \pm} g+i \psi_{ \pm}^{2}\right)
$$

is conserved, while the fermion equations of motion are

$$
\partial_{\mp} \psi_{ \pm}+\frac{1}{2}\left[g^{-1} \partial_{\mp} g, \psi_{ \pm}\right]+\frac{i}{4}\left[\psi_{\mp}^{2}, \psi_{ \pm}\right]=0 .
$$

As in the bosonic case, there can be important discrete symmetries of the SPCM. In particular, we have a $\mathcal{G}$-parity symmetry

$$
\pi: G \mapsto G^{-1} \quad \Rightarrow \quad J_{ \pm} \mapsto-G J_{ \pm} G^{-1}
$$

The derivatives $D_{ \pm} J_{ \pm}$do not have definite behaviour under $\pi$, so we introduce instead the combinations

$$
J_{ \pm \pm}=D_{ \pm} J_{ \pm}+i J_{ \pm}^{2} \quad \Rightarrow \quad J_{ \pm \pm} \mapsto-G J_{ \pm \pm} G^{-1}
$$

Similar modifications can be made to higher derivatives (which proved useful in [2]).

The classical super PCM is superconformally invariant, with the non-vanishing components of the super energy-momentum tensor obeying

$$
D_{-} \operatorname{Tr}\left(J_{+} J_{++}\right)=D_{+} \operatorname{Tr}\left(J_{-} J_{--}\right)=0
$$

When expanded in components this contains conservation equations for both the supersymmetry current and the conventional (bosonic) energy momentum tensor. 


\subsection{Poisson brackets in the SPCM}

Poisson brackets will always be written $\{A, B\}$ but must be understood to be graded, i.e. antisymmetric if either $A$ or $B$ is bosonic, but symmetric if both $A$ and $B$ are fermionic. They obey the Leibnitz rules

$$
\{A, B C\}=\{A, B\} C \pm B\{A, C\}, \quad\{C B, A\}=C\{B, A\} \pm\{C, A\} B
$$

where the minus signs occur if and only if both $A$ and $B$ are fermionic.

The brackets for the bosonic conserved currents (3.21) are

$$
\begin{aligned}
& \left\{j_{0}^{a}(x), j_{0}^{b}(y)\right\}=f^{a b c} j_{0}^{c}(x) \delta(x-y) \\
& \left\{j_{0}^{a}(x), j_{1}^{b}(y)\right\}=f^{a b c} j_{1}^{c}(x) \delta(x-y)+\delta^{a b} \delta^{\prime}(x-y) \\
& \left\{j_{1}^{a}(x), j_{1}^{b}(y)\right\}=-\frac{1}{4} i f^{a b c}\left(h_{+}^{c}(x)+h_{-}^{c}(x)\right) \delta(x-y)
\end{aligned}
$$

or, in light-cone coordinates,

$$
\begin{aligned}
\left\{j_{ \pm}^{a}(x), j_{ \pm}^{b}(y)\right\}= & \frac{1}{2} f^{a b c}\left(3 j_{ \pm}^{c}(x)-j_{\mp}^{c}(x)-\frac{1}{2} i h_{+}^{c}(x)-\frac{1}{2} i h_{-}^{c}(x)\right) \delta(x-y) \\
& \pm 2 \delta^{a b} \delta^{\prime}(x-y) \\
\left\{j_{+}^{a}(x), j_{-}^{b}(y)\right\}= & \frac{1}{2} f^{a b c}\left(j_{+}^{c}(x)+j_{-}^{c}(x)+\frac{1}{2} i h_{+}^{c}(x)+\frac{1}{2} i h_{-}^{c}(x)\right) \delta(x-y)
\end{aligned}
$$

where we have introduced the bosonic quantities

$$
h_{ \pm}=\psi_{ \pm}^{2}, \quad h_{ \pm}^{a}=\frac{1}{2} f^{a b c} \psi_{ \pm}^{b} \psi_{ \pm}^{c} .
$$

The fermions obey

$$
\left\{\psi_{ \pm}^{a}(x), \psi_{ \pm}^{b}(y)\right\}=-i \delta^{a b} \delta(x-y), \quad\left\{\psi_{+}^{a}(x), \psi_{-}^{b}(y)\right\}=0 .
$$

It is also useful to note that

$$
\begin{aligned}
& \left\{h_{ \pm}^{a}(x), \psi_{ \pm}^{b}(y)\right\}=i f^{a b c} \psi_{ \pm}^{c}(x) \delta(x-y) \\
& \left\{h_{ \pm}^{a}(x), h_{ \pm}^{b}(y)\right\}=i f^{a b c} h_{ \pm}^{c}(x) \delta(x-y)
\end{aligned}
$$

Finally, there are non-trivial brackets between the bosonic currents and the fermions

$$
\left\{j_{ \pm}^{a}(x), \psi_{ \pm}^{b}(y)\right\}=\frac{3}{2} f^{a b c} \psi_{ \pm}^{c}(x) \delta(x-y), \quad\left\{j_{ \pm}^{a}(x), \psi_{\mp}^{b}(y)\right\}=\frac{1}{2} f^{a b c} \psi_{\mp}^{c}(x) \delta(x-y)
$$

A derivation of the brackets above is sketched in an appendix, section 6 . 


\subsection{Local conserved charges}

The simplest local conserved currents in the bosonic PCM are powers of the energymomentum tensor (2.20). The super energy-momentum tensor in the SPCM is a fermionic quantity, however, so we cannot take powers of it to obtain new conservation laws in quite the same way. Let us therefore turn directly to the generalisations of (2.22) and (2.19). The currents (2.22) in the bosonic PCM can be generalised to the supersymmetric PCM in two ways. First, we have ${ }^{9}$

$$
D_{-} \operatorname{Tr}\left(J_{+}^{2 n+1}\right)=0
$$

which is odd under the discrete symmetry $\pi$. The power of $J_{+}$must be an odd integer, otherwise the expression would vanish identically, by Fermi statistics. Second, we have

$$
D_{-} \operatorname{Tr}\left(J_{+}^{2 n-1} J_{++}\right)=0
$$

which is even under $\pi$. The power of $J_{+}$must again be odd, this time to prevent the expression being a total $D_{+}$derivative and hence giving a trivial conservation equation. The first member of this sequence, with $n=1$, is the super-energy-momentum tensor. Both (3.31) and (3.32) follow directly from the superspace equations of motion (3.16).

As in the bosonic case, one can generalise these conservation equations by re-writing them in terms of invariant tensors. Equation (3.31) becomes

$$
D_{-}\left(\Omega_{a_{1} a_{2} \ldots a_{2 n+1}} J_{+}^{a_{1}} J_{+}^{a_{2}} \ldots J_{+}^{a_{2 n+1}}\right)=0
$$

where we define, from a symmetric $d$-tensor of rank $n+1$, a completely antisymmetric tensor of rank $2 n+1$ by

$$
\Omega_{a_{1} a_{2} \ldots a_{2 n+1}}=\frac{1}{2^{n}} f_{\left[a_{1} a_{2}\right.}{ }^{b_{1}} \ldots f_{a_{2 n-1} a_{2 n}}^{b_{n}} d^{b_{1} \ldots b_{n}}{ }_{\left.a_{2 n+1}\right]} .
$$

In a similar fashion, the second kind of conservation equation (3.32) becomes

$$
D_{-}\left(\Lambda_{a_{1} \ldots a_{2 n-1} a_{2 n}} J_{+}^{a_{1}} \ldots J_{+}^{a_{2 n-1}} J_{++}^{a_{2 n}}\right)=0
$$

where now the relevant invariant tensor is even-rank,

$$
\Lambda_{a_{1} a_{2} \ldots a_{2 n-1} a_{2 n}}=\frac{1}{2^{n-1}} f_{\left[a_{1} a_{2}\right.}^{b_{1}} \ldots f_{a_{2 n-3} a_{2 n-2}}^{b_{n-1}} d^{\left.b_{1} \ldots b_{n-1} a_{2 n-1}\right] a_{2 n}} .
$$

\footnotetext{
${ }^{9}$ We restrict immediately to positive spins; there are obviously analogous negative-spin currents annihilated by $D_{+}$. See also [15] for a related construction.
} 
It has a more complicated structure in that it is antisymmetric only on its first $2 n-1$ indices.

It is clear that we need invariant tensors which are antisymmetric in some number of indices, in order to combine the fermionic currents $J_{+}$into holomorphic expressions. An immediate consequence of this fact, however, is that there are only finitely many such expressions, in contrast to the infinitely many holomorphic currents (2.19) in the bosonic PCM, which are based on symmetric invariant tensors.

It is useful to pin-point the precise algebraic properties of the $\Omega$ and $\Lambda$ tensors which are relevant here. It can be shown that $\Omega$ vanishes whenever the symmetric tensor $d$ in (3.34) is of compound type. As a result, it is only the primitive part of $d$ which contributes to the expression for $\Omega$ and moreover $\Omega$ is independent (up to an overall factor) of how this primitive tensor is chosen. The situation for $\Lambda$ is only slightly more complicated. If $d$ is a compound tensor made up of just two primitive tensors, then $\Lambda$ does not vanish, but it reduces to a product of two $\Omega$ tensors. If $d$ is compound and made up of three or more primitive tensors, then $\Lambda$ vanishes. These properties are explained in detail in an appendix, section 9 .

To gain a better understanding of the superspace conservation equations, we expand them in component fields, using (3.19). Both kinds of conserved current are holomorphic, and we distinguish their components with superscripts \pm to indicate their behaviour under the $\mathcal{G}$-parity symmetry $\pi$. Thus

$$
\Omega_{a_{1} a_{2} \ldots a_{2 n+1}} J_{+}^{a_{1}} J_{+}^{a_{2}} \ldots J_{+}^{a_{2 n+1}}=\mathcal{F}_{n+\frac{1}{2}}^{-}+(2 n+1) \theta^{+} \mathcal{B}_{n+1}^{-}
$$

(the insertion of the factor $(2 n+1)$ proves convenient) where

$$
\begin{aligned}
\mathcal{F}_{n+\frac{1}{2}}^{-} & =\Omega_{a_{1} a_{2} \ldots a_{2 n+1}} \psi_{+}^{a_{1}} \psi_{+}^{a_{2}} \ldots \psi_{+}^{a_{2 n+1}} \\
& =d_{a_{1} a_{2} \ldots a_{n} a_{n+1}} h_{+}^{a_{1}} h_{+}^{a_{2}} \ldots h_{+}^{a_{n}} \psi_{+}^{a_{n+1}} \\
\mathcal{B}_{n+1}^{-} & =\Omega_{a_{1} \ldots a_{2 n} a_{2 n+1}} \psi_{+}^{a_{1}} \ldots \psi_{+}^{a_{2 n}} j_{+}^{a_{2 n+1}} \\
& =d_{a_{1} a_{2} \ldots a_{n} a_{n+1}} h_{+}^{a_{1}} h_{+}^{a_{2}} \ldots h_{+}^{a_{n}} j_{+}^{a_{n+1}}
\end{aligned}
$$

The expansion of the other currents is more complicated:

$$
\Lambda_{a_{1} \ldots a_{2 n-1} a_{2 n}} J_{+}^{a_{1}} \ldots J_{+}^{a_{2 n-1}} J_{++}^{a_{2 n}}=\mathcal{F}_{n+\frac{1}{2}}^{+}+\theta^{+} \mathcal{B}_{n+1}^{+}
$$

where 


$$
\begin{aligned}
\mathcal{F}_{n+\frac{1}{2}}^{+} & =\Lambda_{a_{1} a_{2} \ldots a_{2 n-1} a_{2 n}} \psi^{a_{1}} \ldots \psi^{a_{2 n-1}} j_{+}^{a_{2 n}}-\frac{1}{2} i \mathcal{F}_{n+\frac{1}{2}}^{-} \\
& =d_{a_{1} a_{2} a_{3} \ldots a_{n+1}} j_{+}^{a_{1}} \psi_{+}^{a_{2}} h_{+}^{a_{3}} \ldots h_{+}^{a_{n+1}}-\frac{1}{2} i \mathcal{F}_{n+\frac{1}{2}}^{-} \\
\mathcal{B}_{n+1}^{+} & =\Lambda_{a_{1} a_{2} \ldots a_{2 n-1} a_{2 n}}\left((2 n-1) j_{+}^{a_{1}} j_{+}^{a_{2 n}}+i \psi_{+}^{a_{1}} \partial_{+} \psi_{+}^{a_{2 n}}\right) \psi_{+}^{a_{2}} \ldots \psi_{+}^{a_{2 n-1}}-(n-1) i \mathcal{B}_{n+1}^{-} \\
& =d_{a_{1} a_{2} a_{3} \ldots a_{n+1}}\left(n j_{+}^{a_{1}} j_{+}^{a_{2}}+i \psi_{+}^{a_{1}} \partial_{+} \psi_{+}^{a_{2}}\right) h_{+}^{a_{3}} \ldots h_{+}^{a_{n+1}}-(n-1) i \mathcal{B}_{n+1}^{-}
\end{aligned}
$$

(recall the bosonic quantity $h_{+}^{a}=\frac{1}{2} f^{a b c} \psi_{+}^{b} \psi_{+}^{c}$ ).

In either family of conservation laws, the fermionic and bosonic currents have spins $n+\frac{1}{2}$ and $n+1$ respectively, and so the corresponding conserved charges have spins $n-\frac{1}{2}$ and $n$ respectively. The fact that the currents are based on a primitive $d$-tensor of rank $n+1$ then implies that the values of $n$ are precisely the exponents of the algebra.

Little can be said at present about the effects of quantisation on these conservation equations, although counting arguments can be used to demonstrate the persistence of some of them [2], following the methods of [22, 23]. What is certain, however, is that (super)conformal invariance will be broken in passing from the classical to the quantum SPCM, and so one cannot expect the conservation equations to survive in (super)holomorphic form. Now we have already seen that a typical non-holomorphic superspace conservation equation (3.1) leads to a conserved bosonic charge without, in general, any fermionic partner. This suggests that while the fermionic currents $\mathcal{F}^{ \pm}$are relevant to understanding the classical structure of the SPCM, it is the bosonic currents $\mathcal{B}^{ \pm}$which are of more central importance in the quantum theory.

A last word of caution should be added. It is quite possible for a pair of bosonic and fermionic charges to arise from a non-holomorphic conservation law, and a familiar example is provided by energy-momentum and its fermionic partner, supersymmetry. The classical holomorphic equation (3.23) for the super-energy-momentum tensor will certainly receive quantum modifications, and yet we expect that supersymmetry will also survive in the quantum theory. Such behaviour is only possible when the non-holomorphic superspace current has a very particular structure. For the case of the super-energy-momentum tensor, this special structure is easily understood (as a consequence of Noether's Theorem) and this is explained in an appendix, section 7. We can see no reason to expect similar behaviour for the superspace conservation equations of higher spin. 


\subsection{Comparing the bosonic and supersymmetric PCMs}

Essentially by construction, the super PCM reduces to the bosonic PCM when all fermions are set to zero. The formulas for the conserved currents such as (2.22) and (3.31) seem very similar superficially, but here the superspace notation and the fermionic character of the current in the SPCM hide some profound differences. In the bosonic PCM there are infinitely many holomorphic quantities (2.19). In the supersymmetric PCM we found two distinct sets (3.33) and (3.35), each of them containing only finitely many holomorphic quantities. Notice also that when we reduce the SPCM to the bosonic PCM by setting the fermions to zero, all the currents $\mathcal{B}^{ \pm}$and $\mathcal{F}^{ \pm}$vanish, with the exception of $\mathcal{B}_{2}^{+}$(which is of course the ++ component of the energy-momentum tensor). This begs a question: Are there other conserved quantities in the SPCM which will reduce to any of the currents in (2.19) when the fermions vanish? We will argue that there are not, but to do so we must consider what possible forms such conservation equations might take, so as to be able to phrase the question more precisely.

First observe that the series of holomorphic quantities $\operatorname{Tr}\left(j_{+}^{m}\right)$ in the bosonic PCM relies on the form of the equation of motion of the current: $\partial_{-} j_{+}=($const. $)\left[j_{+}, j_{-}\right]$. It is actually irrelevant for this purpose that $j_{ \pm}$is conserved. All that is important is that $\partial_{-} j_{+}$can be expressed as a commutator of $j_{+}$with some other field in the Lie algebra. In the SPCM, the bosonic current $j_{ \pm}$is defined by (3.21). Any quantity of the form $k_{+}=j_{+}+\alpha \psi_{+}^{2}$ will reduce to the conserved current component in the bosonic PCM when the fermions are set to zero, and this is obviously the most general such polynomial in the current and the fermions with spin 1. A precise way to pose the question of the last paragraph is now to ask whether it is possible to find $k_{-}=j_{-}+\beta \psi_{-}^{2}$ such that

$$
\partial_{-} k_{+}=\gamma\left[k_{-}, k_{+}\right]
$$

in the SPCM. This would be enough to ensure the existence of holomorphic quantities $\operatorname{Tr}\left(k_{+}^{m}\right)$ in the SPCM of the type we seek. After a short calculation, however, one finds that such an equation implies an over-determined set of relations amongst the constants $\alpha, \beta$ and $\gamma$, and there is no solution. Details of a more general calculation which includes the effects of a WZ term are given in the next section.

One might object that the form assumed in (3.43) is already too restrictive. We can avoid this assumption and still carry out a similar argument if we focus on the simplest example of currents of spin 3 (in the SPCM based on $s u(N)$ for example). Thus, we can 
consider the most general $\left(\mathcal{G}_{L} \times \mathcal{G}_{R}\right)$-invariant polynomial in the fields $j_{+}$and $\psi_{+}$with this spin, and ask whether it can ever be holomorphic. It actually suffices to consider

$$
\operatorname{Tr}\left(j_{+}^{3}\right)+\alpha \operatorname{Tr}\left(j_{+}^{2} \psi_{+}^{2}\right)
$$

since the only other possible terms are $\operatorname{Tr}\left(j_{+} \psi_{+} j_{+} \psi_{+}\right)=0$ identically, by Fermi statistics and cyclicity of the trace, and $\operatorname{Tr}\left(j_{+} \psi_{+}^{4}\right)$, which we already know to be holomorphic, and which is therefore of no help in achieving our goal. Once again an explicit calculation reveals that there is no choice of $\alpha$ for which the expression above is holomorphic (see also the following section).

These results may seem unexpected, but in fact similar behaviour is known to occur in other integrable models and their supersymmetric extensions. Specifically, for bosonic conformal Toda theories, and their $(1,0)$ supersymmetric extensions [26], one finds [27] that the higher-spin conserved quantities generating the $\mathcal{W}$-algebra of the bosonic theory have no generalisations (in a suitably precise sense, similar to the ones above). Here, as there, it would appear that an 'interference' arises between supersymmetry and the existence of currents with non-trivial spin. The ultimate implications for these models are rather different however. In the (1,0) Toda theories, no other conserved quantities are known, and it was conjectured on this basis that these supersymmetric extensions are not integrable [27]. In the SPCM there are higher-spin local (as well as non-local) conserved quantities which guarantee integrability. As we have seen, these are genuinely new features of the SPCM in the sense that they do not reduce to the familiar conserved quantities of the bosonic PCM when the fermions are set to zero.

\subsection{The effect of a WZ term and the special nature of the WZW points}

So far we have dealt exclusively with the SPCM, allowing us to keep the presentation as simple as possible. We now discuss briefly the modifications which arise on adding a WZ term, which can easily be constructed in superspace, as in e.g. [20]. The effect of a suitably normalised WZ term with coefficient $\lambda$ is to modify the equation obeyed by the currents $J_{ \pm}=-i G^{-1} D_{ \pm} G$ so that it becomes

$$
(1-\lambda) D_{+} J_{-}-(1+\lambda) D_{-} J_{+}=0
$$

Adopting the same component field definitions as before, we find, after eliminating the auxiliary field,

$$
(1+\lambda) \partial_{-} j_{+}+(1-\lambda) \partial_{+} j_{-}=0 \quad \text { where } \quad j_{ \pm}=-g^{-1} \partial_{ \pm} g-i \psi_{ \pm}^{2}
$$


and

$$
\partial_{\mp} \psi_{ \pm}+\frac{1}{2}(1 \mp \lambda)\left[g^{-1} \partial_{\mp} g, \psi_{ \pm}\right]+\frac{i}{4}\left(1-\lambda^{2}\right)\left[\psi_{\mp}^{2}, \psi_{ \pm}\right]=0 .
$$

The construction of the two sets of superfield currents in (3.33) and (3.35) is completely unaffected, no matter what the value of $\lambda$.

Some important qualitative differences arise at the critical values of the coupling $\lambda=$ \pm 1 which define super WZW theories. For $\lambda=1$, for instance, the current becomes super-holomorphic: $D_{-} J_{+}=0$. This means of course that there is a simplified superfield expansion $J_{+}=\psi_{+}+\theta^{+} j_{+}$with $\partial_{-} \psi_{+}=\partial_{-} j_{+}=0$. These holomorphic equations of motion arise in conjunction with the super Kac-Moody symmetry of the critical theory [20, 21].

Earlier we stated that there was no natural way to generalise the local conservation laws of the bosonic PCM to the SPCM by using an equation such as (3.43), or by looking for a holomorphic quantity such as (3.44). These same calculations can be carried out more generally in the PCWZM and its supersymmetric counterpart, and it is instructive to elaborate on the details.

First we use (3.46) and (3.47) to find

$$
\partial_{-} j_{+}=-\frac{1}{2}(1-\lambda)\left[j_{+}, j_{-}\right]-\frac{i}{4}(1-\lambda)^{2}\left[j_{+}, \psi_{-}^{2}\right]+\frac{i}{4}\left(1-\lambda^{2}\right)\left[j_{-}, \psi_{+}^{2}\right]+\frac{1}{4}(1-\lambda)\left(1-\lambda^{2}\right)\left[\psi_{+}^{2}, \psi_{-}^{2}\right]
$$

Now we apply this expression together with (3.47) to calculate both sides of

$$
\partial_{-} k_{+}=\gamma\left[k_{-}, k_{+}\right] \quad \text { where } \quad k_{+}=j_{+}+i \alpha \psi_{+}^{2}, \quad k_{-}=j_{-}+i \beta \psi_{-}^{2} .
$$

Comparing coefficients of like terms on the left- and right-hand sides gives the relations

$$
\begin{aligned}
\gamma & =\frac{1}{2}(1-\lambda) \\
\beta \gamma & =\frac{1}{4}(1-\lambda)^{2} \\
\alpha \gamma & =\frac{1}{2} \alpha(1-\lambda)+\frac{1}{4}\left(1-\lambda^{2}\right) \\
\alpha \beta \gamma & =\frac{1}{2} \alpha(1-\lambda)+\frac{1}{4}\left(1-\lambda^{2}\right)(1-\lambda-\alpha)
\end{aligned}
$$

Substituting for $\gamma$ from the first equation into the third reveals that the equations are consistent only for $\lambda^{2}=1$, the super WZW points. Thus if $\lambda \neq \pm 1$, including the super PCM with $\lambda=0$, then there is no relation of the type we seek.

If $\lambda=1$, the general solution is $\gamma=0$, with $\alpha, \beta$ arbitrary. We then recover from (3.49) the familiar conditions $\partial_{-} j_{+}=\partial_{-} \psi_{+}=0$. If $\lambda=-1$, the general solution is $\gamma=\beta=1$ 
and $\alpha$ arbitrary. This is also as expected, because at this second WZW point it is the left-transforming quantities $g j_{+} g^{-1}$, and $g \psi_{+} g^{-1}$ which should be holomorphic, and thus each of them should satisfy $\partial_{-}\left(g X g^{-1}\right)=0$, or equivalently, $\partial_{-} X+\left[g^{-1} \partial_{-} g, X\right]=0$, which is indeed the content of (3.49). The solutions for $\lambda= \pm 1$ are therefore less surprising than the lack of solutions for $\lambda \neq \pm 1$.

In a similar fashion, we can examine the possibility of a holomorphic quantity of the form (3.44). Using (3.48) and (3.47) it is a simple matter to calculate $\partial_{-} \operatorname{Tr}\left(j_{+}^{3}\right)$ and $\partial_{-} \operatorname{Tr}\left(j_{+}^{2} \psi_{+}^{2}\right)$. Both expressions have an overall factor of $\left(\lambda^{2}-1\right)$, and the coefficients of like terms cannot be matched except when this vanishes. Thus, supersymmetry does not allow a generalisation of such a spin-3 current, except in the super WZW models.

We drew attention previously to the fact that the bosonic WZW theory (with $\lambda=1$ say) contains an enlarged set of holomorphic currents polynomial in $j_{+}^{a}$. Similarly, for the super WZW theory (with $\lambda=1$ ) the currents (3.33) and (3.35) exist within a much larger set of holomorphic quantities consisting of arbitrary polynomials in both $j_{+}^{a}$ and $\psi_{+}^{a}$. The holomorphic currents in the bosonic WZW theory obviously extend immediately to the super WZW theory. This simple relationship between these enlarged sets of conserved quantities in the critical theories is of course responsible for the solutions to (3.49) found at $\lambda= \pm 1$. By contrast, our results indicate that there is no simple connection between the conserved quantities in the PCWZM and its super-extension at non-critical coupling $\lambda \neq \pm 1$.

\subsection{Non-local charges}

The non-local charges for a supersymmetric sigma model may be constructed [24] in component formalism using a rather complicated generalisation of the iterative procedure described earlier for the bosonic case. The result is again $Y_{L} \times Y_{R}$ : there are no new fermionic superpartners for the charges. The construction looks neater in the superfield formalism [25], where it is a natural extension of the bosonic case, and the lack of superpartners is accounted for by the general discussion we gave in section 3. Here we include the effect of a superspace $\mathrm{WZ}$ term, $\lambda \neq 0$.

We define a superspace connection acting on any bosonic quantity $X$ in the Lie algebra (if $X$ is fermionic we replace the commutator with an anti-commutator):

$$
\nabla_{ \pm} X=(1 \pm \lambda)\left(D_{ \pm} X+i\left[J_{ \pm}, X\right]\right) \Rightarrow\left\{\nabla_{+}, \nabla_{-}\right\}=0
$$


by virtue of (3.15). From this we can define an infinite family of superfield currents $J_{ \pm}^{(r)}$ for $r=0,1,2 \ldots$ which will be conserved:

$$
D_{-} J_{+}^{(r)}-D_{+} J_{-}^{(r)}=0 \quad \Leftrightarrow \quad J_{ \pm}^{(r)}= \pm D_{ \pm} X^{(r)}
$$

for some scalar superfields $X^{(r)}$. The first two currents are

$$
J_{ \pm}^{(0)}=(1 \pm \lambda) J_{ \pm}, \quad J_{ \pm}^{(1)}=(1 \pm \lambda)\left(D_{ \pm} X^{(0)}-\frac{1}{2}\left[J_{ \pm}, X^{(0)}\right]\right)
$$

whose conservation follows from (3.45) and (3.15). The remaining currents are defined by (3.50) and

$$
J_{ \pm}^{(r)}=\nabla_{ \pm} X^{(r-1)} \quad r>1
$$

It is easy to prove by induction that these are conserved: if this holds for all $r \leq n$ then

$$
\begin{aligned}
D_{-} J_{+}^{(n+1)}-D_{+} J_{-}^{(n+1)} & =\left(D_{-} \nabla_{+}-D_{+} \nabla_{-}\right) X^{(n)} \\
& =-\left(\nabla_{+} D_{-}-\nabla_{-} D_{+}\right) X^{(n)} \\
& =\nabla_{+} J_{-}^{(n)}+\nabla_{-} J_{+}^{(n)} \\
& =\left\{\nabla_{+}, \nabla_{-}\right\} X^{(n-1)} \quad(n>1) \\
& =0 .
\end{aligned}
$$

The corresponding non-local conserved charges are given by

$$
\begin{aligned}
Q^{(n)} & =\int\left(d x^{+} d \theta_{+} J_{+}^{(n)}-d x^{-} d \theta_{-} J_{-}^{(n)}\right) \\
& =\int\left(d x^{+} j_{+}^{(n)}-d x^{-} j_{-}^{(n)}\right)+\theta^{-} \int d x^{+} \partial_{+} \alpha_{-}^{(n)}-\theta^{+} \int d x^{-} \partial_{-} \alpha_{+}^{(n)}
\end{aligned}
$$

and it may be checked that $\alpha_{ \pm}^{(n)} \rightarrow 0$ as $x \rightarrow \pm \infty$. (Our notation for current components was introduced in (3.2).) The first two examples are

$$
\begin{aligned}
Q^{(0) a}= & \int d x j_{0}^{(0) a}(x) \\
Q^{(1) a}= & \int d x\left(j_{1}^{(0) a}(x)+\lambda j_{0}^{(0) a}(x)+\frac{i}{2}\left((1-\lambda)^{2} h_{-}^{a}-(1+\lambda)^{2} h_{+}^{a}\right)\right. \\
& \left.-\frac{1}{2} f^{a b c} \int^{x} d y j_{0}^{(0) b}(x) j_{0}^{(0) c}(y)\right) .
\end{aligned}
$$

When $\lambda=0$ we make the link with the first paper of [24] by pointing out that, comparing with their eqns. $(2.12,3.9)$, their $B_{ \pm}$equals our $\frac{1}{2} i h_{ \pm}$.

When $\lambda=0$ it is a straightforward though cumbersome calculation to show that the non-local charges defined above commute with all four sets of local charges obtained from (3.38-3.42); see the appendix, section 8. We have not attempted this calculation for $\lambda \neq 0$. 


\section{Classical current algebra and commuting local charges in the SPCM}

In the last section we constructed local, holomorphic superspace currents (3.33) and (3.35) in the classical SPCM. Each such current gave a pair of fermionic and bosonic holomorphic currents $\mathcal{F}_{n+1 / 2}^{ \pm}$and $\mathcal{B}_{n+1}^{ \pm}$in ordinary space. Our aim now is to analyse and understand the properties of these currents at a level comparable to our treatment of the bosonic PCWZM in section 2. Thus our first goal will be to compute the Poisson bracket (PB) algebra of a certain class of currents. Using these results, we will then search for commuting sets of charges.

To carry out the Poisson bracket calculations, it proves convenient to introduce the modified currents

$$
\hat{\jmath}_{+}=j_{+}+\frac{3}{2} i h_{+}, \quad \hat{\jmath}_{-}=j_{-}+\frac{1}{2} i h_{+}+\frac{1}{2} i h_{-}
$$

By construction, these have vanishing PBs with the fermions $\psi_{+}$. They can be shown to obey

$$
\begin{aligned}
& \left\{\hat{\jmath}_{+}^{a}(x), \hat{\jmath}_{+}^{b}(y)\right\}=\frac{1}{2} f^{a b c}\left(3 \hat{\jmath}_{+}^{c}(x)-\hat{\jmath}_{-}^{c}(x)\right) \delta(x-y)+2 \delta^{a b} \delta^{\prime}(x-y) \\
& \left\{\hat{\jmath}_{+}^{a}(x), \hat{\jmath}_{-}^{b}(y)\right\}=\frac{1}{2} f^{a b c}\left(\hat{\jmath}_{+}^{c}(x)+\hat{\jmath}_{-}^{c}(x)+\frac{1}{2} i h_{-}^{c}(x)\right) \delta(x-y) .
\end{aligned}
$$

We will also need the fermion equation of motion

$$
\partial_{-} \psi_{+}=\frac{1}{2}\left[\hat{\jmath}_{-}, \psi_{+}\right] \quad \Rightarrow \quad \partial_{+} \psi_{+}=2 \psi^{\prime}+\frac{1}{2}\left[\hat{\jmath}_{-}, \psi_{+}\right]
$$

to express all currents in terms of good canonical variables, involving only space derivatives of the fermions.

\subsection{Poisson bracket algebra for currents built from symmetric traces}

Following the same route as for the bosonic PCM, we shall investigate currents built from symmetric-trace type invariants. In principle all others can be expressed in terms of these (albeit in a rather inconvenient, non-polynomial way for the case of the Pfaffian invariant). After substituting in favour of the modified quantities just introduced, the odd-parity currents are:

$$
\begin{aligned}
\mathcal{F}_{m+\frac{1}{2}}^{-} & =s_{a_{1} \ldots a_{m} a_{m+1}} h_{+}^{a_{1}} \ldots h_{+}^{a_{m}} \psi_{+}^{a_{m+1}}=\operatorname{Tr}\left(\psi_{+}^{2 m+1}\right) \\
\mathcal{B}_{m+1}^{-} & =s_{a_{1} \ldots a_{m} a_{m+1}} h_{+}^{a_{1}} \ldots h_{+}^{a_{m}} \hat{\jmath}_{+}^{a_{m+1}}=\operatorname{Tr}\left(\psi_{+}^{2 m} \hat{\jmath}_{+}\right) .
\end{aligned}
$$


The even-parity currents are considerably more complicated:

$$
\begin{aligned}
& \mathcal{F}_{m+\frac{1}{2}}^{+}=s_{a_{1} \ldots a_{m} a_{m+1}} h_{+}^{a_{1}} \ldots h_{+}^{a_{m}}\left(\hat{\jmath}_{+}^{a_{m+1}}-\frac{1}{2} i \psi_{+}^{a_{m+1}}\right)=\operatorname{Tr}\left(\psi_{+}^{2 m-1} \hat{\jmath}_{+}\right)-\frac{i}{2} \mathcal{F}_{m+\frac{1}{2}}^{-} \\
& \mathcal{B}_{m+1}^{+}=s_{a_{1} \ldots a_{m} a_{m+1}}\left(2 i \psi_{+}^{a_{1}} \psi_{+}^{\prime a_{2}}-i h_{+}^{a_{1}} \hat{\jmath}_{-}^{a_{2}}+m \hat{\jmath}_{+}^{a_{1}} \hat{\jmath}_{+}^{a_{2}}-(m-1) i h_{+}^{a_{1}} \hat{\jmath}_{+}^{a_{2}}\right) h_{+}^{a_{3}} \ldots h_{+}^{a_{m+1}} \\
& =2 i \operatorname{Tr}\left(\psi_{+}^{2 m-1} \psi_{+}^{\prime}\right)-i \operatorname{Tr}\left(\psi_{+}^{2 m} \hat{\jmath}_{-}\right)+\sum_{r=0}^{m-1} \operatorname{Tr}\left(\psi_{+}^{2 r} \hat{\jmath}_{+} \psi_{+}^{2 m-2-2 r} \hat{\jmath}_{+}\right)-(m-1) i \mathcal{B}_{m+1}^{-}
\end{aligned}
$$

To calculate the PBs of these currents one can begin in the obvious way, by repeated application of the Leibnitz rules. The resulting expressions can be simplified by the use of completeness conditions in the relevant Lie algebra. In particular, if $X$ is any element of $s o(N)$ or $s p(N)$ then $X^{m}$ also belongs to the Lie algebra when $m$ is odd, and hence $\operatorname{Tr}\left(X^{m} t^{c}\right) \operatorname{Tr}\left(Y t^{c}\right)=-\operatorname{Tr}\left(X^{m} Y\right)$ for any $Y$. The algebra $s u(N)$ works a little differently, since the matrix must be traceless in order to apply the completeness condition. In this case we can write instead $\operatorname{Tr}\left(X^{m} t^{c}\right)=\operatorname{Tr}\left(\left(X^{m}-\frac{1}{N} \operatorname{Tr}\left(X^{m}\right) 1\right) t^{c}\right)$ and then $\operatorname{Tr}\left(X^{m} t^{c}\right) \operatorname{Tr}\left(Y t^{c}\right)=$ $-\operatorname{Tr}\left(X^{m} Y\right)+\frac{1}{N} \operatorname{Tr} X^{m} \operatorname{Tr} Y$ for any integer $m$. To obtain the results given below it is also necessary to take particular care with Fermi statistics and combinatoric factors, and to make use of the cyclic properties of the trace to show that certain terms vanish.

The simplest brackets to calculate are those of the odd-parity currents amongst themselves, which can be shown to vanish:

$$
\begin{array}{r}
\left\{\mathcal{F}_{m+\frac{1}{2}}^{-}(x), \mathcal{F}_{n+\frac{1}{2}}^{-}(y)\right\}=0 \\
\left\{\mathcal{F}_{m+\frac{1}{2}}^{-}(x), \mathcal{B}_{n+1}^{-}(y)\right\}=0 \\
\left\{\mathcal{B}_{m+1}^{-}(x), \mathcal{B}_{n+1}^{-}(y)\right\}=0
\end{array}
$$

For the odd-parity with even-parity currents we find:

$$
\begin{aligned}
\left\{\mathcal{F}_{m+\frac{1}{2}}^{-}(x), \mathcal{F}_{n+\frac{1}{2}}^{+}(y)\right\} & =(2 m+1) i \mathcal{B}_{m+n}^{-} \delta(x-y) \\
\left\{\mathcal{B}_{m+1}^{-}(x), \mathcal{F}_{n+\frac{1}{2}}^{+}(y)\right\} & =-2 \mathcal{F}_{m+n-\frac{1}{2}}^{-} \delta^{\prime}(x-y)-\frac{2(2 n-1)}{2 m+2 n-1} \mathcal{F}_{m+n-\frac{1}{2}}^{-\prime} \delta(x-y) \\
\left\{\mathcal{F}_{m+\frac{1}{2}}^{-}(x), \mathcal{B}_{n+1}^{+}(y)\right\} & =-2(2 m+1) \mathcal{F}_{m+n-\frac{1}{2}}^{-} \delta^{\prime}(x-y)-\frac{4 n(2 m+1)}{2 m+2 n-1} \mathcal{F}_{m+n-\frac{1}{2}}^{-\prime} \delta(x-y) \\
\left\{\mathcal{B}_{m+1}^{-}(x), \mathcal{B}_{n+1}^{+}(y)\right\} & =-4(m+n) \mathcal{B}_{m+n}^{-} \delta^{\prime}(x-y)-4 n \mathcal{B}_{m+n}^{-\prime} \delta(x-y)
\end{aligned}
$$

where for clarity we have omitted the argument $x$ from all currents appearing on the righthand side. The most difficult brackets to calculate are those of the even parity currents with themselves. After some effort we obtain the results: 


$$
\begin{aligned}
& \left\{\mathcal{F}_{m+\frac{1}{2}}^{+}(x), \mathcal{F}_{n+\frac{1}{2}}^{+}(y)\right\}=i \mathcal{B}_{m+n}^{+} \delta(x-y)+\frac{1}{N}\left[-i \mathcal{B}_{m}^{-} \mathcal{B}_{n}^{-}+2 \mathcal{F}_{m-\frac{1}{2}}^{-} \mathcal{F}_{n-\frac{1}{2}}^{-\prime}\right] \delta(x-y) \\
& +\frac{2}{N} \mathcal{F}_{m-\frac{1}{2}}^{-} \mathcal{F}_{n-\frac{1}{2}}^{-} \delta^{\prime}(x-y) \\
& \left\{\mathcal{F}_{m+\frac{1}{2}}^{+}(x), \mathcal{B}_{n+1}^{+}(y)\right\}=-2(2 m+2 n-1) \mathcal{F}_{m+n-\frac{1}{2}}^{+} \delta^{\prime}(x-y)-4 n \mathcal{F}_{m+n-\frac{1}{2}}^{+\prime} \delta(x-y) \\
& +\frac{4 n}{N}\left[\frac{1}{2 n-1} \mathcal{B}_{m}^{-} \mathcal{F}_{n-\frac{1}{2}}^{-\prime}+\mathcal{F}_{m-\frac{1}{2}}^{-} \mathcal{B}_{n}^{-\prime}\right] \delta(x-y) . \\
& +\frac{1}{N}\left[2 \mathcal{B}_{m}^{-} \mathcal{F}_{n-\frac{1}{2}}^{-}+4 n \mathcal{F}_{m-\frac{1}{2}}^{-} \mathcal{B}_{n}^{-}\right] \delta^{\prime}(x-y) \\
& \left\{\mathcal{B}_{m+1}^{+}(x), \mathcal{B}_{n+1}^{+}(y)\right\}=-4(m+n) \mathcal{B}_{m+n}^{+} \delta^{\prime}(x-y)-4 n \mathcal{B}_{m+n}^{+\prime} \delta(x-y) \\
& +\frac{1}{N}\left[8 n m \mathcal{B}_{m}^{-} \mathcal{B}_{n}^{-\prime}-\frac{8 n i}{(2 n-1)(2 m-1)} \mathcal{F}_{m-\frac{1}{2}}^{-\prime} \mathcal{F}_{n-\frac{1}{2}}^{-\prime}+\frac{8 n i}{2 n-1} \mathcal{F}_{m-\frac{1}{2}}^{-} \mathcal{F}_{n-\frac{1}{2}}^{-\prime \prime}\right] \delta(x-y) \\
& +\frac{1}{N}\left[8 n m \mathcal{B}_{m}^{-} \mathcal{B}_{n}^{-}-\frac{4 i}{2 m-1} \mathcal{F}_{m-\frac{1}{2}}^{-\prime} \mathcal{F}_{n-\frac{1}{2}}^{-}+\frac{4 i(4 n-1)}{2 n-1} \mathcal{F}_{m-\frac{1}{2}}^{-} \mathcal{F}_{n-\frac{1}{2}}^{-\prime}\right] \delta^{\prime}(x-y) \\
& +\frac{4 i}{N} \mathcal{F}_{m-\frac{1}{2}}^{-} \mathcal{F}_{n-\frac{1}{2}}^{-} \delta^{\prime \prime}(x-y)
\end{aligned}
$$

Once again, all fields on the right-hand side are at argument $x$. The terms with $1 / N$ coefficients occur only for the algebra $s u(N)$, since for the other algebras $m$ and $n$ are always odd.

Important consistency checks of these complicated calculations come from supersymmetry. The current $\mathcal{F}_{3 / 2}^{+}$is precisely the Noether current for supersymmetry, and so

$$
\int d x\left\{\mathcal{F}_{3 / 2}^{+}, X\right\}
$$

where $X$ is some current $\mathcal{F}_{n+1 / 2}^{ \pm}$or $\mathcal{B}_{n+1}^{ \pm}$, must reproduce the transformations (3.10) applied to these quantities. (In comparing the results one should remember that $\partial_{-} \alpha=0$ implies $\partial_{+} \alpha=2 \alpha^{\prime}$.) An even more powerful restriction arises if we take $X$ to be a Poisson bracket of currents. Let us suppose (using an obvious notation) that we have calculated a $\mathrm{PB}$ of the form $\left\{\mathcal{F}_{1}(x), \mathcal{F}_{2}(y)\right\}$. We can work out its variation under supersymmetry directly, but by the Leibnitz rule for PBs this is also proportional to the combination $\left\{\mathcal{B}_{1}(x), \mathcal{F}_{2}(y)\right\}-\left\{\mathcal{F}_{1}(x), \mathcal{B}_{2}(y)\right\}$, giving a non-trivial relationship between the latter two brackets. Similarly, if we have calculated $\left\{\mathcal{B}_{1}(x), \mathcal{F}_{2}(y)\right\}$, we can apply supersymmetry to relate $\left\{\mathcal{B}_{1}(x), \mathcal{B}_{2}(y)\right\}$ to $\left\{\mathcal{F}_{1}^{\prime}(x), \mathcal{F}_{2}(y)\right\}$. All the results above are consistent with such considerations. 


\subsection{Commuting charges - by direct calculation}

We will now search for commuting sets of bosonic conserved charges, beginning from

$$
B_{n}^{-}=\int d x \mathcal{B}_{n+1}^{-}(x), \quad B_{n}^{+}=\int d x \mathcal{B}_{n+1}^{+}(x)
$$

based on the symmetric trace invariants used in the current algebra above.

There are a number of reasons why it seems natural not to consider fermionic charges in the same way. For one thing, we explained in the last section that it is quite possible for a bosonic charge to survive quantisation without being accompanied by a superpartner. Even if a fermionic charge were to survive quantisation along with its bosonic partner, it is not clear that it is very interesting to find 'commuting' sets. This is because 'commuting' for these classical charges really means 'vanishing graded Poisson brackets', and if such an algebra is unmodified quantum-mechanically, the fermionic charges will obey $F^{2}=0$. In a quantum theory with a positive-definite Hilbert space, such charges can only be represented trivially. One might then be prompted to consider other possibilities for the fermionic charge algebra, with $F^{2} \neq 0$, and indeed such behaviour is evident already at the classical level in the PBs of the currents $\mathcal{F}_{n+1 / 2}^{+}$. These are in some sense higher-spin versions of supersymmetry, with $F^{2} \sim B$ (schematically). While such possibilities are certainly interesting, they lie beyond our immediate goals in this paper.

Let us turn then to the PB algebra of the bosonic charges which is easily found to be

$$
\begin{aligned}
& \left\{B_{m}^{-}, B_{n}^{-}\right\}=0 \\
& \left\{B_{m}^{-}, B_{n}^{+}\right\}=0 \\
& \left\{B_{m}^{+}, B_{n}^{+}\right\}=\frac{8 m n}{N} \int d x\left(\mathcal{B}_{m}^{-} \mathcal{B}_{n}^{-\prime}-\frac{2 i}{(2 m-1)(2 n-1)} \mathcal{F}_{m-\frac{1}{2}}^{-\prime} \mathcal{F}_{n-\frac{1}{2}}^{-\prime}\right)
\end{aligned}
$$

The terms on the right-hand-side vanish for the algebras $s o(N)$ and $s p(N)$, though not for $s u(N)$. This is highly reminiscent of the problems we were faced with in the bosonic theory. A further similarity is that our discussion so far is based on trace-type invariants, and so omits the Pfaffian in $s o(2 \ell)$.

Taking the same approach as before, we will first search for modifications of the evenparity currents which will yield commuting charges for $\mathbf{g}=s u(N)$. The simplest possibility

is to add terms bilinear in currents $\mathcal{B}_{m}^{-}$and $\mathcal{F}_{m-1 / 2}^{-}$, since these will naturally provide contributions with the same structure as the unwanted terms we are hoping to cancel. In 
addition the trivial PBs of the negative parity currents implies that such modifications will not spoil the desirable property that the second bracket in (4.10) vanishes.

Starting from a general ansatz, one finds after some algebra that the new currents

$$
\mathcal{K}_{m+1}^{+}=\mathcal{B}_{m+1}^{+}-\frac{m}{N} \sum_{p=2}^{m-1} \mathcal{B}_{p}^{-} \mathcal{B}_{m-p+1}^{-}-\frac{m}{N} \sum_{p=2}^{m-1} \frac{2 i}{(2 p-1)(2 m-2 p+1)} \mathcal{F}_{p-\frac{1}{2}}^{-} \mathcal{F}_{m-p+\frac{1}{2}}^{-\prime}
$$

have exactly the desired properties: i.e. the corresponding charges $K_{n}^{+}=\int d x \mathcal{K}_{n+1}^{+}$obey

$$
\left\{B_{m}^{-}, B_{n}^{-}\right\}=\left\{B_{m}^{-}, K_{n}^{+}\right\}=\left\{K_{m}^{+}, K_{n}^{+}\right\}=0 .
$$

These equations represent a highly over-determined system of conditions for the coefficients of the new terms, so it is quite non-trivial that these have the correct properties. In fact the result applies not just for $s u(N)$, but also for $s o(N)$ and $s p(N)$, with the $1 / N$ in the formulas above replaced by an arbitrary real number. This further reinforces the analogy with the bosonic case.

It is natural to ask whether the additional terms correspond to anything simple in terms of the invariant tensors underlying the conserved currents. The change from $\mathcal{B}_{m+1}^{+}$to $\mathcal{K}_{m+1}^{+}$ actually amounts to a replacement

$$
\Lambda_{a_{1} \ldots a_{2 m}}^{(2 m)} \rightarrow \Lambda_{a_{1} \ldots a_{2 m}}^{(2 m)}-\frac{1}{N} \sum_{p=2}^{m-1} \Omega_{\left[a_{1} \ldots a_{2 p-1}\right.}^{(2 p-1)} \Omega_{\left.a_{2 p} \ldots a_{2 m-1}\right] a_{2 m}}^{(2 m-2 p+1)}
$$

which is easily checked for the fermionic terms in (4.11) (up to some irrelevant total derivatives) and which can be verified for the bosonic modifications too. Using detailed relationships between the invariant tensors which are derived in one of the appendices, section 9 , this is found to correspond to a replacement of the underlying symmetric tensor

$$
s_{a_{1} \ldots a_{m+1}}^{(m+1)} \rightarrow k_{a_{1} \ldots a_{m+1}}^{(m+1)},
$$

precisely the set introduced in our treatment of the bosonic PCWZM in section 2.

While this may seem very satisfactory, we must emphasise that the $k$-tensors were introduced in the bosonic theory to simplify the algebra of charges resulting from (2.29). A priori, there is no reason to expect such a direct link with the considerably more complicated current algebra (4.9) and it is therefore puzzling why the $k$-tensors should provide the required simplification in the SPCM too. In the next section we shall resolve this puzzle by establishing a link with the earlier, bosonic, current algebra. This approach also has the advantage of working for a general invariant tensor, so that the Pfaffian charge in $s o(2 \ell)$ can be treated in exactly the same way as the other primitive invariants. 


\subsection{Commuting charges - by comparison with bosonic PCM}

It is convenient to modify our notation very slightly. We now take $k_{a_{1} a_{2} \ldots a_{n} a_{n+1}}$ to be any of the symmetric invariant tensors introduced in section 2 via (2.33), or the Pfaffian invariant in $s o(2 \ell)$ (previously written $p$ in $(2.25)$ ). This means that the $k$-tensors are now a complete set of primitive invariants for any algebra. Now denote the bosonic currents (3.39) and (3.42) with $d^{(n+1)}=k^{(n+1)}$, by $\mathcal{K}_{n}^{-}$and $\mathcal{K}_{n}^{+}$respectively. The corresponding charges will be written

$$
K_{n}^{-}=\int d x \mathcal{K}_{n+1}^{-}(x)=\int d x k_{a_{1} a_{2} \ldots a_{n+1}} \hat{\jmath}_{+}^{a_{1}} h_{+}^{a_{2}} \ldots h_{+}^{a_{n+1}}
$$

and

$$
K_{n}^{+}=\int d x \mathcal{K}_{n+1}^{+}(x)=U_{n}+V_{n}+W_{n}-i(n-1) K_{n}^{-}
$$

where

$$
\begin{aligned}
U_{n} & =2 i \int d x k_{a_{1} a_{2} \ldots a_{n+1}} \psi_{+}^{a_{1}} \psi_{+}^{a_{2} \prime} h_{+}^{a_{3}} \ldots h_{+}^{a_{n+1}}, \\
V_{n} & =n \int d x k_{a_{1} a_{2} \ldots a_{n+1}} \hat{\jmath}_{+}^{a_{1}} \hat{\jmath}_{+}^{a_{2}} h_{+}^{a_{3}} \ldots h_{+}^{a_{n+1}}, \\
W_{n} & =-i \int d x k_{a_{1} a_{2} \ldots a_{n+1}} \hat{\jmath}_{-}^{a_{1}} h_{+}^{a_{2}} \ldots h_{+}^{a_{n+1}} .
\end{aligned}
$$

(Since the $\Omega$ tensors are unique, $K_{n}^{-}$is identical to $B_{n}^{-}$of the last subsection when the underlying $k$-tensor is not the Pfaffian.) We will prove below that these charges commute:

$$
\left\{K_{m}^{-}, K_{n}^{-}\right\}=\left\{K_{m}^{-}, K_{n}^{+}\right\}=\left\{K_{m}^{+}, K_{n}^{+}\right\}=0
$$

One useful approach to these rather complicated calculations is to introduce the quantities

$$
\mathcal{H}^{a}=h_{+}^{a}+\alpha \hat{\jmath}_{+}^{a} \quad \text { and } \quad \tilde{\mathcal{H}}^{a}=h_{+}^{a}+\beta \hat{\jmath}_{-}^{a}
$$

and to observe that

$$
\begin{aligned}
K_{n}^{-} & =\left.\frac{1}{n+1} \int d x k_{a_{1} a_{2} \ldots a_{n+1}} \mathcal{H}^{a_{1}} \mathcal{H}^{a_{2}} \ldots \mathcal{H}^{a_{n+1}}\right|_{\alpha} \\
V_{n} & =\left.\frac{2}{n+1} \int d x k_{a_{1} a_{2} \ldots a_{n+1}} \mathcal{H}^{a_{1}} \mathcal{H}^{a_{2}} \ldots \mathcal{H}^{a_{n+1}}\right|_{\alpha^{2}} \\
W_{n} & =-\left.\frac{i}{n+1} \int d x k_{a_{1} a_{2} \ldots a_{n+1}} \tilde{\mathcal{H}}^{a_{1}} \tilde{\mathcal{H}}^{a_{2}} \ldots \tilde{\mathcal{H}}^{a_{n+1}}\right|_{\beta}
\end{aligned}
$$

This provides a convenient way of handling the various combinatorial issues which arise. Furthermore, the PB algebra of the new currents has a structure similar to that encountered 
in the bosonic PCM:

$$
\begin{aligned}
\left\{\mathcal{H}^{a}(x), \mathcal{H}^{b}(y)\right\} & =f^{a b c}\left(i h_{+}^{c}+\frac{1}{2} \alpha^{2}\left(3 \hat{\jmath}_{+}^{c}-\hat{\jmath}_{-}^{c}\right)\right) \delta(x-y)+2 \alpha^{2} \delta^{a b} \delta^{\prime}(x-y) \\
\left\{\mathcal{H}^{a}(x), \tilde{\mathcal{H}}^{b}(y)\right\} & =f^{a b c}\left(i h_{+}^{c}+\frac{1}{2} \alpha \beta\left(\hat{\jmath}_{+}^{c}+\hat{\jmath}_{-}^{c}\right)+\frac{1}{4} \alpha \beta i h_{-}^{c}\right) \delta(x-y)
\end{aligned}
$$

The simplest computation is the bracket of two odd-parity charges, $K_{m}^{-}$and $K_{n}^{-}$, which is proportional to

$$
\left.\left\{\int d x k_{a_{1} a_{2} \ldots a_{m+1}} \mathcal{H}^{a_{1}} \mathcal{H}^{a_{2}} \ldots \mathcal{H}^{a_{m+1}}, \int d y k_{b_{1} b_{2} \ldots b_{n+1}} \mathcal{H}^{b_{1}} \mathcal{H}^{b_{2}} \ldots \mathcal{H}^{b_{n+1}}\right\}\right|_{\alpha^{2}}
$$

The only surviving contribution is

$$
\int d x k_{a_{1} a_{2} \ldots a_{m} c}^{(m+1)} h_{+}^{a_{1}} h_{+}^{a_{2}} \ldots h_{+}^{a_{m}} k_{b_{1} \ldots b_{n-1} b_{n} c}^{(n+1)} h_{+}^{b_{1}} \ldots h_{+}^{b_{n-1}} h_{+}^{b_{n} \prime}
$$

but this integrand vanishes due to invariance of the $k$-tensors, taken together with the fact that $h_{+}^{a}=\frac{1}{2} f^{a b c} \psi_{+}^{b} \psi_{+}^{c}$. Indeed, if the expression is re-written in terms of $\Omega$ tensors, its vanishing is equivalent to the identity

$$
\Omega_{c\left[a_{1} \ldots a_{2 m}\right.}^{(2 m+1)} \Omega_{\left.b_{1} \ldots b_{2 n-1}\right] b_{2 n} c}^{(2 n+1)}=0
$$

(This can be proved by writing the $\Omega$-tensors as in (9.8) and using the invariance condition (9.2).) Thus $\left\{K_{m}^{-}, K_{n}^{-}\right\}=0$, as claimed.

Turning next to the bracket of $K_{m}^{-}$with $K_{n}^{+}$, it suffices to consider the brackets of the odd-parity charge with $U_{n}, V_{n}$ and $W_{n}$. For the first of these, we need a lemma:

$$
\left\{U_{n}, h_{+}^{a}(x)\right\}=\left\{U_{n}, \mathcal{H}^{a}(x)\right\}=-4\left(k_{a_{1} \ldots a_{n} a} h_{+}^{a_{1}} \ldots h_{+}^{a_{n}}\right)^{\prime}(x)
$$

Then from (4.14) we find

$$
\left\{K_{m}^{-}, U_{n}\right\}=\int d x 4 m n k_{a_{1} \ldots a_{m-1} a_{m} c}^{(m+1)} h_{+}^{a_{1}} \ldots h_{+}^{a_{m-1}} \hat{\jmath}_{+}^{a_{m}} k_{b_{1} \ldots b_{n-1} b_{n} c}^{(n+1)} h_{+}^{b_{1}} \ldots h_{+}^{b_{n-1}} h_{+}^{b_{n} \prime} .
$$

The remaining brackets we need are

$$
\begin{aligned}
\left\{K_{m}^{-}, V_{n}\right\}= & -\int d x 4 m n k_{a_{1} \ldots a_{m-1} a_{m} c}^{(m+1)} h_{+}^{a_{1}} \ldots h_{+}^{a_{m-1}} h_{+}^{a_{m} \prime} k_{b_{1} \ldots b_{n-1} b_{n} c}^{(n+1)} h_{+}^{b_{1}} \ldots h_{+}^{b_{n-1}} \hat{\jmath}_{+}^{b_{n}} \\
& +\int d x n k_{a_{1} \ldots a_{m} a}^{(m+1)} h_{+}^{a_{1}} \ldots h_{+}^{a_{m}} k_{b_{1} \ldots b_{n-1} b_{n} b}^{(n+1)} h_{+}^{b_{1}} \ldots h_{+}^{b_{n-1}} \hat{\jmath}_{+}^{b_{n}} f^{a b c} \hat{\jmath}_{-}^{c}
\end{aligned}
$$

which follows from (4.14) and (4.15), and

$$
\left\{K_{m}^{-}, W_{n}\right\}=-\int d x m n k_{a_{1} \ldots a_{m-1} a_{m} a}^{(m+1)} h_{+}^{a_{1}} \ldots h_{+}^{a_{m-1}} \hat{\jmath}_{+}^{a_{m}} k_{b_{1} \ldots b_{n-1} b_{n} b}^{(n+1)} h_{+}^{b_{1}} \ldots h_{+}^{b_{n-1}} \hat{\jmath}_{-}^{b_{n}} f^{a b c} h_{+}^{c}
$$


which follows from (4.14) and (4.16). In each of these calculations it is necessary to make extensive use of the invariance conditions for the $k$-tensors. These same conditions then imply that the total contribution is

$$
\left\{K_{m}^{-}, K_{n}^{+}\right\}=\left\{K_{m}^{-}, U_{n}\right\}+\left\{K_{m}^{-}, V_{n}\right\}+\left\{K_{m}^{-}, W_{n}\right\}=0
$$

Finally we come to the lengthiest calculation: the bracket of two even-parity charges. Given the properties of the odd-parity charges, it suffices to consider the brackets of the quantities $U, V$ and $W$ amongst themselves, presenting us with six different expressions to evaluate. Some of these are very similar to the calculations we have already sketched above. In particular, we find $\left\{W_{m}, W_{n}\right\}=\left\{U_{m}, W_{n}\right\}+\left\{W_{m}, U_{n}\right\}=0$. The non-trivial contributions can then be usefully divided into two: the terms

$$
\left\{V_{m}, V_{n}\right\}+\left\{U_{m}, V_{n}\right\}+\left\{V_{m}, U_{n}\right\}+\left\{W_{m}, V_{n}\right\}+\left\{V_{m}, W_{n}\right\}
$$

which can be treated using the formulas (4.15) and (4.16) together with the lemma introduced earlier; and a single remaining term $\left\{U_{m}, U_{n}\right\}$ which must be evaluated by other means. We will now show that both sets of terms vanish, by comparing with the known results for the bosonic models.

After some work it can be shown that (4.18) is equal to

$$
\left.\frac{4}{(m+1)(n+1)}\left\{\int d x k_{a_{1} a_{2} \ldots a_{m+1}} \mathcal{H}^{a_{1}} \mathcal{H}^{a_{2}} \ldots \mathcal{H}^{a_{m+1}}, \int d y k_{b_{1} b_{2} \ldots b_{n+1}} \mathcal{H}^{b_{1}} \mathcal{H}^{b_{2}} \ldots \mathcal{H}^{b_{n+1}}\right\}\right|_{\alpha^{4}}
$$

This expression obviously contains one of the desired brackets, $\left\{V_{m}, V_{n}\right\}$, but it also generates other terms which turn out to match exactly the remaining contributions in (4.18). Now we need only compare the current algebra (4.17) of the $\mathcal{H}^{a}$ with (2.17) in the bosonic PCM to understand why this expression vanishes. Exactly the same arguments (as given in section 2 and [1]) ensure that the ultralocal terms will not contribute, and so we have the same charge algebra as for the bosonic PCM, up to an overall constant arising from the coefficients of the $\delta^{\prime}$ terms. The tensors $k$ were chosen precisely to ensure that the charge PBs vanished in the bosonic PCM. Hence (4.18) also vanishes.

To complete the computation of the even-parity charge brackets it remains to consider

$$
\left\{U_{m}, U_{n}\right\}=-16 m n i \int d x k_{a_{1} \ldots a_{m-1} b c}^{(m+1)} k_{a_{m} \ldots a_{m+n-2} d c}^{(n+1)} h_{+}^{a_{1}} \ldots h_{+}^{a_{m+n-2}} \psi_{+}^{\prime b} \psi_{+}^{\prime d},
$$

which is once again arrived at by extensive use of invariance conditions. The antisymmetry in $b$ and $d$ imposed by $\psi_{+}^{\prime} b \psi_{+}^{\prime} d$ allows us to write this, up to a factor, as

$$
\int d x k_{\left(a_{1} \ldots a_{m-1} b\right.}^{(m+1)}{ }^{c} k_{\left.a_{m} \ldots a_{m+n-2}\right) d c}^{(n+1)} h_{+}^{a_{1}} \ldots h_{+}^{a_{m+n-2}} \psi_{+}^{\prime b} \psi_{+}^{\prime d} .
$$


But now recall that the vital property of the $k$-tensors that guarantees commuting charges in the bosonic PCM can be expressed as (2.39). This immediately implies that the bracket $\left\{U_{m}, U_{n}\right\}$ vanishes.

We have now established (4.13). Notice that most of the arguments - including all those underlying the vanishing of the PBs of the odd-parity charges - did not involve any special property of the $k$-tensors (beyond their invariance). The special nature of the $k$ tensors was used at precisely two points above in showing that the even-parity charges have vanishing PBs too. In making comparisons with the current algebra of the bosonic PCM, we have clarified why the same tensors arise in the SPCM.

\section{$5 \quad$ Summary and conclusions}

In the bosonic PCWZM, there are infinitely many holomorphic conservation laws (2.19) based on symmetric invariant tensors. From amongst this set, it is possible to define commuting local charges based on the particular symmetric tensors $k$ defined in section 2 . There is an infinite sequence for each primitive invariant, with spins repeating modulo the Coxeter number of the algebra. All this is completely independent of the coefficient of the WZ term.

In the supersymmetric versions of these $\operatorname{models}^{10}$, there are finitely many independent holomorphic conservation laws $(3.33,3.35)$. As explained in section 3, they are based on antisymmetric invariant tensors, which can nevertheless be related to symmetric primitive invariants in the algebra. This leads to bosonic conserved charges with spins exactly equal to the exponents, but with no repetition modulo the Coxeter number. These charges commute with one another when the symmetric invariants are chosen to be precisely the same tensors $k$ that arose in the bosonic theory.

There is no direct relationship between the currents $(2.19)$ and $(3.33,3.35)$ and in fact the latter vanish when the fermions are set to zero. A simple and direct relationship exists only between much larger sets of holomorphic currents which are special features of the WZW and super WZW models. A rather subtle indirect relationship can be established between the underlying current algebras (2.29) and (4.7)-(4.9), however, which explains the importance of the same set of tensors $k$ for both the bosonic and supersymmetric theories.

\footnotetext{
${ }^{10}$ We discussed mainly the SPCM, but the extension to the supersymmetric PCWZM should be obvious in view of our detailed treatment of the bosonic theories.
} 


\section{Acknowledgments}

We thank Jose Azcárraga, Patrick Dorey and Gérard Watts for discussions. The research of JME is supported by a PPARC Advanced Fellowship, and by NSF grant PHY98-02484. NJM thanks Pembroke College Cambridge for a Stokes Fellowship, during which early stages of this work were carried out. MH is grateful to St. John's College, Cambridge for a Studentship. AJM thanks the 1851 Royal Commission for a Research Fellowship.

\section{Appendix: Poisson brackets in the PCWZM and SPCM}

A general $\sigma$-model with WZ term can be described by a lagrangian of the form

$$
\begin{aligned}
\mathcal{L}= & \frac{1}{2} g_{i j}(\phi) \partial_{\mu} \phi^{i} \partial^{\mu} \phi^{j}+\frac{1}{2} b_{i j}(\phi) \varepsilon^{\mu \nu} \partial_{\mu} \phi^{i} \partial_{\nu} \phi^{j} \\
& =\frac{1}{2} g_{i j}(\phi)\left(\dot{\phi}^{i} \dot{\phi}^{j}-\phi^{\prime i} \phi^{\prime j}\right)+b_{i j}(\phi) \dot{\phi}^{i} \phi^{\prime j}
\end{aligned}
$$

where $\phi^{i}$ are coordinates on some target manifold equipped with a metric $g_{i j}(\phi)$ and antisymmetric tensor field $b_{i j}(\phi)$. The momenta conjugate to the fields $\phi^{i}$ are

$$
\pi_{i}=\hat{\pi}_{i}+b_{i j} \phi^{j} \quad \text { where } \quad \hat{\pi}_{i}=g_{i j} \dot{\phi}^{j}
$$

by definition. These obey the standard non-vanishing equal-time PBs

$$
\left\{\phi^{i}(x), \pi_{j}(y)\right\}=\delta^{i}{ }_{j} \delta(x-y)
$$

A short calculation reveals that

$$
\left\{\hat{\pi}_{i}(x), \hat{\pi}_{j}(y)\right\}=h_{i j k} \phi^{\prime k} \delta(x-y) \quad \text { where } \quad h_{i j k}=\partial_{i} b_{j k}+\partial_{j} b_{k i}+\partial_{k} b_{i j}
$$

Now consider a (non-conserved) current

$$
E_{\mu}^{a}=E_{i}^{a} \partial_{\mu} \phi^{i}
$$

where $E_{i}^{a}(\phi)$ are vielbeins on the target manifold satisfying

$$
E_{i}^{a} E_{j}^{a}=g_{i j}
$$

In terms of the canonical coordinates $\phi^{i}$ and $\pi_{i}$ we have

$$
E_{0}^{a}=E_{i}^{a} g^{i j} \hat{\pi}_{j}, \quad E_{1}^{a}=E_{i}^{a} \phi^{\prime i}
$$


The PB algebra of these currents can now be calculated routinely, although the general result requires some effort and is not particularly illuminating.

Important simplification occurs for the special case of a group manifold, with currents defined by the (right-transforming) vielbeins

$$
E_{i}^{a}=\operatorname{Tr}\left(t^{a} g^{-1} \partial_{i} g\right), \quad \text { obeying } \quad \partial_{[i} E_{j]}=E_{[i} E_{j]} .
$$

Let us also choose the WZ term to be related to the structure constants

$$
h^{i j k} E_{i}^{a} E_{j}^{b} E_{k}^{c}=-\lambda f^{a b c}
$$

with $\lambda$ some constant. This is precisely the PCWZM defined, in different notation, in the main text. For this case, the results of the current algebra calculations simplify to give

$$
\begin{aligned}
& \left\{E_{0}^{a}(x), E_{0}^{b}(y)\right\}=f^{a b c}\left(E_{0}^{c}-\lambda E_{1}^{c}\right) \delta(x-y) \\
& \left\{E_{0}^{a}(x), E_{1}^{b}(y)\right\}=f^{a b c} E_{1}^{c} \delta(x-y)+\delta^{a b} \delta^{\prime}(x-y) \\
& \left\{E_{1}^{a}(x), E_{1}^{b}(y)\right\}=0
\end{aligned}
$$

Notice that the only effect of the WZ term is the contribution proportional to the constant $\lambda$. Translating to light-cone components gives

$$
\begin{aligned}
& \left\{E_{ \pm}^{a}(x), E_{ \pm}^{b}(y)\right\}=\frac{1}{2} f^{a b c}\left((3 \mp \lambda) E_{ \pm}^{c}-(1 \mp \lambda) E_{\mp}^{c}\right) \delta(x-y) \pm 2 \delta^{\prime}(x-y) \\
& \left\{E_{+}^{a}(x), E_{-}^{b}(y)\right\}=\frac{1}{2} f^{a b c}\left((1-\lambda) E_{+}^{c}+(1+\lambda) E_{-}^{c}\right) \delta(x-y)
\end{aligned}
$$

In the PCWZM the conserved current has components $j_{ \pm}=j_{0} \pm j_{1}=(1 \pm \lambda)\left(E_{0} \pm E_{1}\right)=$ $(1 \pm \lambda) E_{ \pm}$. The expressions given in the main text, with $\kappa=1$, now follow immediately.

Now we consider the supersymmetric PCM, using the same notation for coordinates $\phi^{i}$ and vielbeins $E_{i}^{a}$ on the group as above, but with fermions $\psi_{ \pm}^{a}$ valued in the Lie-algebra (i.e. carrying tangent-space indices) as in the main text. To determine the Poisson brackets, only the terms in the lagrangian involving time derivatives of the fields are important. After re-writing the couplings $\operatorname{Tr}\left(\psi_{ \pm}\left[g^{-1} \partial_{\mp} g, \psi_{ \pm}\right]\right)$in coordinate notation, the only relevant terms are

$$
\frac{1}{2} g_{i j}(\phi) \dot{\phi}^{i} \dot{\phi}^{j}+\frac{i}{2} \psi_{+} \dot{\psi}_{+}+\frac{i}{2} \psi_{-} \dot{\psi}_{-}+\frac{i}{2} E_{j}^{a} \dot{\phi}^{j}\left(h_{+}^{a}+h_{-}^{a}\right)
$$

The brackets amongst the fermions are just those of a free theory, with the standard normalizations for real fermions. Moreover, they have vanishing brackets with the fields $\phi^{i}$ and with their conjugate momenta

$$
\pi_{i}=\hat{\pi}_{i}+\frac{i}{2} E_{i}^{a}\left(h_{+}^{a}+h_{-}^{a}\right) \quad \text { where } \quad \hat{\pi}_{i}=g_{i j} \dot{\phi}^{j}
$$


The conserved currents in the SPCM have spacetime components

$$
\begin{aligned}
j_{0}^{a} & =E_{i}^{a} \dot{\phi}^{i}-\frac{i}{2}\left(h_{+}^{a}+h_{-}^{a}\right)=E^{a i} \pi_{i}-i\left(h_{+}^{a}+h_{-}^{a}\right) \\
j_{1}^{a} & =E_{i}^{a} \phi^{i}-\frac{i}{2}\left(h_{+}^{a}-h_{-}^{a}\right)
\end{aligned}
$$

It is now straightforward to calculate the algebra by comparing with the result for the bosonic PCM $(\lambda=0)$ above, and using the results (3.28) for the fermions. One finds (3.24) together with

$$
\left\{j_{0}^{a}(x), \psi_{ \pm}^{b}(y)\right\}=f^{a b c} \psi_{ \pm}^{c} \delta(x-y), \quad\left\{j_{1}^{a}(x), \psi_{ \pm}^{b}(y)\right\}= \pm \frac{1}{2} f^{a b c} \psi_{ \pm}^{c} \delta(x-y) .
$$

Changing to light-cone components gives the expressions quoted in the text.

By combining the approaches above, the Poisson brackets of the super PCWZM can be calculated in a similar fashion.

\section{Appendix: Conservation laws in superspace}

The superspace conservation equation (3.1) has component content (3.3)-(3.6). Only the first of these equations represents a conservation law, in general. For the special case of a holomorphic current, however, there is an additional conserved quantity as in (3.9) and the pair are related by supersymmetry (3.11). The first issue we wish to clarify here is how such a superpartner can arise in more general circumstances, including necessary and sufficient conditions for this to happen.

In order for (3.4) to give an additional conservation law we require that $\beta_{-}=-\partial_{+} \omega_{---}$ for some (spin-3/2) fermion $\omega_{---}$, so that

$$
\partial_{-} \alpha_{+}+\partial_{+} \omega_{---}=0 \quad \Rightarrow \quad F^{+}=\int\left(d x^{+} \alpha_{+}+d x^{-} \omega_{---}\right)
$$

is a new conserved charge, which is related to $B$ in (3.7) by supersymmetry. But by applying a supersymmetry transformation, we find that the constraint $\beta_{-}=-\partial_{+} \omega_{---}$is consistent only if $v=-\partial_{+} k_{-}$, for some (spin-1) boson $k_{-}$. Taken together, these imply

$$
J_{-}=-i D_{+} K_{-}, \quad \text { where } \quad K_{-}=k_{-}+i \theta^{+} \alpha_{-}+i \theta^{-} \omega_{---}+i \theta^{+} \theta^{-} j_{-} .
$$

Thus a necessary and sufficient condition for the existence of a conserved charge $F^{+}$whose variation under $Q_{+}$gives $B$, is that we can write $J_{-}=-i D_{+} K_{-}$for some superfield $K_{-}$. 
The holomorphic case corresponds to the simplest possibility $K_{-}=0$. There is also the independent possibility that we can construct a superpartner charge $F^{-}$related to $B$ by $Q_{-}$, which arises if and only if $J_{+}=-i D_{-} K_{+}$for some superfield $K_{+}$.

Notice that when (7.2) is satisfied, we can re-express (3.1) in the form

$$
D_{-}\left(i \theta^{+} J_{+}\right)-D_{+}\left(i \theta^{+} J_{-}-K_{-}\right)=0 \text {. }
$$

This is also a superspace conservation equation, but the current components are not superfields. We can construct a conserved quantity from this new equation by using the standard formulas in (3.7), and the result is $F^{+}$. Our previous arguments ensuring invariance under supersymmetry of the conserved charge $B$ do not apply to $F^{+}$, because the current components in (7.3) involve $\theta$ explicitly.

It is helpful to compare this with symmetries in ordinary (non-super) spacetime. Any charge constructed entirely from fields, such as a momentum generator or an internal symmetry generator, must commute with translations. But charges which involve explicit dependence on $x$-coordinates, such as Lorentz generators, will not commute with translations. Similarly, in superspace, any charge constructed entirely from superfields will necessarily commute with supersymmetry. But charges involving explicit $\theta$-dependence will not.

The second issue we would like to elaborate on is how this discussion applies to energymomentum and supersymmetry. As a consequence of translation invariance, Noether's Theorem guarantees the existence of a superfield conservation law of the general form (3.1) with the bosonic charge $B$ being energy-momentum along some particular direction. For a supersymmetric theory we know there is a conserved superpartner $F$, namely a supersymmetry generator. But to establish this we must also apply Noether's theorem to supersymmetry transformations. Once this is done, we find that the definition of the translation superfield current can indeed always be improved so as to fulfill the condition (7.2), in accordance with our general results.

The necessity of carrying out such an improvement in the conformal case was discussed in [23], but in language pre-dating the modern development of conformal field theory. In contemporary terminology, this is simply the statement that in a superconformal field theory we can always improve the canonical super-energy-momentum tensor so that its conservation becomes a holomorphic conservation equation. To complete our discussion we will show how this improvement works in a general supersymmetric theory, whether 
conformal or not.

In the main text we deliberately avoided the raising and lowering of spinor indices. Now it will be more helpful to allow this possibility. We shall distinguish vector indices $\mu, \nu, \ldots$ and spinor indices $a, b, \ldots$ with the summation convention applied to all contracted upper and lower indices. The rule for raising and lowering spinor indices is $F^{ \pm}= \pm F_{\mp}$. Thus, for example, the standard superspace current conservation equation reads

$$
D_{a} J^{a}=D_{+} J^{+}+D_{-} J^{-}=D_{+} J_{-}-D_{-} J_{+}=0 .
$$

Consider a field theory in superspace, described by a superfield lagrangian $\mathcal{L}\left(\Phi, D_{a} \Phi\right)$. Under the action of graded transformations which change the superfield by $\delta \Phi\left(x^{\mu}, \theta^{a}\right)$, one finds that, on using the equations of motion, the variation of the lagrangian can be expressed in the form

$$
D_{a}\left(\frac{\partial \mathcal{L}}{\partial D_{a} \Phi} \delta \Phi\right)-\delta \mathcal{L}=0
$$

Now the condition for invariance of the action is

$$
\delta \mathcal{L}=D_{a} X^{a} \quad \Rightarrow \quad D_{a} J^{a}=0, \quad \text { with } \quad J^{a}=\frac{\partial \mathcal{L}}{\partial D_{a} \Phi} \delta \Phi-X^{a},
$$

where the first equation defines $X^{a}$. This is the superspace form of Noether's Theorem.

Applying this to $x$-translations in the direction labelled $\mu$, gives

$$
D_{a} T_{\mu}^{a}=0
$$

with $T_{\mu}^{a}$ a vector-spinor superfield. In detail:

$$
D_{a}\left(\frac{\partial \mathcal{L}}{\partial D_{a} \Phi} \partial_{ \pm} \Phi\right)-D_{ \pm}\left(i D_{ \pm} \mathcal{L}\right)=0
$$

where we have made use of the superspace algebra $D_{ \pm}^{2}=-i \partial_{ \pm}$. Applying Noether's Theorem to a supersymmetry labelled by a spinor index $b$, we find

$$
D_{a} S^{a}{ }_{b}=0
$$

where $S^{a}{ }_{b}$ is a spinor-spinor superfield. By making repeated use of the fact that $Q_{ \pm}=$ $D_{ \pm}+2 i \theta^{ \pm} \partial_{ \pm}$we can write the current

$$
S^{a}{ }_{ \pm}=K^{a}{ }_{ \pm}-2 i \theta^{ \pm} T^{a}{ }_{ \pm}, \quad \text { where } \quad K^{a}{ }_{b}=\frac{\partial \mathcal{L}}{\partial\left(D_{a} \Phi\right)} D_{b} \Phi+\delta_{b}^{a} \mathcal{L}
$$


Comparing (7.5) and (7.4) we see that

$$
T_{ \pm}^{ \pm}=-\frac{i}{2} D_{a} K_{ \pm}^{a}
$$

and it is this which allows us to improve the energy-momentum tensor in the way that we desire. For example, considering translations or supersymmetries given by $\mu=b=+$, it follows from (7.6) that we can define an improved superfield current:

$$
\tilde{T}_{+}^{ \pm}=T_{+}^{ \pm}+\frac{i}{2} D_{\mp} K^{-}+
$$

which satisfies

$$
D_{a} \tilde{T}_{+}^{a}=0, \quad \tilde{T}_{+}^{+}=-\frac{i}{2} D_{+} K^{+}+
$$

The last equation exactly meets the criterion (7.2). Similarly, for translations and supersymmetries given by $\mu=b=-$ we have the independent improvement

$$
\tilde{T}_{-}^{ \pm}=T_{-}^{ \pm}+\frac{i}{2} D_{\mp} K^{+}
$$

which satisfies

$$
D_{a} \tilde{T}_{-}^{a}=0, \quad \tilde{T}_{-}^{-}=-\frac{i}{2} D_{-} K_{-}^{-} .
$$

The superpartner charges which arise are of course exactly the supersymmetry generators.

\section{Appendix : Commutation of local with non-local charges}

In this appendix we give some details of the vanishing of the Poisson brackets of the local with the non-local charges for the $\operatorname{SPCM}(\lambda=0)$. Recall that the odd-parity charges $F_{m-\frac{1}{2}}^{-}$ and $B_{m}^{-}$are integrals of the currents

$$
\Omega_{a_{1} a_{2} \ldots a_{2 m+1}} \psi_{+}^{a_{1}} \psi_{+}^{a_{2}} \ldots \psi_{+}^{a_{2 m+1}}, \quad \Omega_{a_{1} \ldots a_{2 m} a_{2 m+1}} \psi_{+}^{a_{1}} \ldots \psi_{+}^{a_{2 m}} j_{+}^{a_{2 m+1}}
$$

respectively. Once we have proved that the non-local charges commute with these, we may prove commutation for the even-parity local charges $F_{m-\frac{1}{2}}^{+}$and $B_{m}^{+}$by considering the densities

$$
d_{a_{1} a_{2} a_{3} \ldots a_{m+1}} j_{+}^{a_{1}} \psi_{+}^{a_{2}} h_{+}^{a_{3}} \ldots h_{+}^{a_{m+1}}, \quad d_{a_{1} a_{2} a_{3} \ldots a_{m+1}}\left(m j_{+}^{a_{1}} j_{+}^{a_{2}}+i \psi_{+}^{a_{1}} \partial_{+} \psi_{+}^{a_{2}}\right) h_{+}^{a_{3}} \ldots h_{+}^{a_{m+1}},
$$

(since they differ by terms proportional to the odd-parity charges). Recalling the definitions $h_{ \pm}=\psi_{ \pm}^{2}$ given in the text, we shall also write $h_{0}=\frac{1}{2}\left(h_{+}+h_{-}\right)$and $h_{1}=\frac{1}{2}\left(h_{+}-h_{-}\right)$. 
It is useful to introduce quantities $b_{\mu}$ by writing the conserved current components in the $\mathrm{SPCM}$

$$
j_{0}=b_{0}-2 i h_{0}, \quad j_{1}=b_{1}-i h_{1}
$$

The quantities $b_{\mu}$ satisfy the same Poisson brackets as the currents in the bosonic PCM $((2.16,2.17)$ with $\lambda=0)$, while the relations (3.28) can also conveniently be re-written

$$
\left\{h_{\mu}^{a}(x), h_{\nu}^{b}(y)\right\}=\frac{1}{2} i f^{a b c} h_{|\mu-\nu|}^{c}(x) \delta(x-y) .
$$

for $\mu, \nu=0,1$.

Since all the local charges we have constructed are singlets in the Lie algebra, the charge $Q^{(0) a}$ must commute with them; this is also relatively simple to check directly. It therefore remains to calculate the brackets with the first non-local charge, which can be written

$$
Q^{(1) a}=\int\left\{b_{1}^{a}(x)-2 i h_{1}^{a}(x)-\frac{1}{2} f^{a b c} j_{0}^{b}(x) \int^{x} j_{0}^{c}(y) d y\right\} d x .
$$

In the calculations which follow, we shall use square brackets to indicate the contributions arising from each of the three terms in the formula for $Q^{(1) a}$ above.

The simplest bracket

$$
\left\{F_{m-\frac{1}{2}}^{-}, Q^{(1) a}\right\}=0
$$

can be calculated quite straightforwardly: the first term is trivially zero, and the other two vanish by invariance of $\Omega$.

Next we consider the even-parity fermionic charge, constructed using a $\Lambda$ tensor which is antisymmetric on all but one of its $2 m$ indices.

$$
\begin{aligned}
\left\{F_{m-\frac{1}{2}}^{+}, Q^{(1) c}\right\}= & \left\{\int \Lambda_{a_{1} a_{2} \ldots a_{2 m-1} b} \psi_{+}^{a_{1}} \psi_{+}^{a_{2}} \ldots \psi_{+}^{a_{2 m-1}} j_{+}^{b} d x, Q^{(1) c}\right\} \\
= & \int d x \Lambda_{a_{1} a_{2} \ldots a_{2 m-1} b}\left\{\left(f^{a_{1} c d} \psi_{+}^{d} \psi_{+}^{a_{2}} \ldots \psi_{+}^{a_{2 m-1}}+\ldots+f^{a_{m-1} c d} \psi_{+}^{a_{1}} \ldots \psi_{+}^{a_{2 m-2}} \psi_{+}^{d}\right) j_{+}^{b}\right. \\
& \left.\quad+f^{b c d} \psi_{+}^{a_{1}} \psi_{+}^{a_{2}} \ldots \psi_{+}^{a_{2 m-1}}\left(\left[b_{1}^{d}\right]+\left[-2 i h_{1}^{d}-i h_{0}^{d}\right]+\left[b_{0}^{d}-2 i h_{0}^{d}\right]\right)\right\} \\
\propto & \int d x \Lambda_{a_{1} a_{2} \ldots a_{2 m-1} b} f^{b c d} \psi_{+}^{a_{1}} \psi_{+}^{a_{2}} \ldots \psi_{+}^{a_{2 m-1}} h_{+}^{d} \\
\propto & \int d x f^{b c d} f^{d a_{2 m} a_{2 m+1}} \Lambda_{a_{1} a_{2} \ldots a_{2 m-1} b} \psi_{+}^{a_{1}} \psi_{+}^{a_{2}} \ldots \psi_{+}^{a_{2 m+1}}
\end{aligned}
$$

But, by the Jacobi identity and invariance:

$$
f^{b c d} f_{[e a}^{d} \Lambda_{\left.a_{1} a_{2} \ldots a_{2 m-1}\right]^{b}}=2 f_{[e}^{c d} f_{a}^{d b} \Lambda_{\left.a_{1} \ldots a_{2 m-1}\right]}{ }^{b}=-2 f_{\left[e^{c d} \Omega^{d}{ }_{\left.a a_{1} \ldots a_{2 m-1}\right]}=0\right.}
$$


Now we move on to the rather tougher bosonic charges. (One could deduce that these commute with $Q^{(1) a}$ by applying supersymmetry to the fermionic results; we shall calculate them directly.) To simplify the results it is wise to introduce some notation in advance. Since invariance of the $d$ tensors plays such an important role, we introduce the short-hand

$$
\underline{A} B C \ldots D \equiv f^{b a_{1} c} d_{a_{1} a_{2} a_{3} \ldots a_{m+1}} A^{c} B^{a_{2}} C^{a_{3}} \ldots D^{a_{m+1}},
$$

Then, by symmetry of $d, \underline{A} B C \ldots D=B \underline{A} C \ldots D=B C \underline{A} \ldots D$ etc. The fact that $d$ is invariant may now be expressed:

$$
\mathbf{d}(A B C \ldots D) \equiv \underline{A} B C \ldots D+A \underline{B} C \ldots D+\ldots+A B C \ldots \underline{D}=0 .
$$

Consider now the odd-parity bosonic charge:

$$
\begin{array}{rl}
\left\{Q^{(1) b}, B_{m}^{-}\right\}=\int d & d d_{a_{1} a_{2} \ldots a_{m+1}}\left\{\left[f^{b a_{1} c} b_{1}^{c} h_{+}^{a_{2}} \ldots h_{+}^{a_{m+1}}\right]\right. \\
+ & {\left[-i f_{b a_{1} c}\left(2 h_{1}^{c}+h_{0}^{c}\right) h_{+}^{a_{2}} \ldots h_{+}^{a_{m+1}}\right.} \\
\quad & \left.+j_{+}^{a_{1}}\left(f^{b a_{2} c} h_{+}^{c} h_{+}^{a_{3}} \ldots h_{+}^{a_{m+1}}+\ldots+f^{b a_{m+1} c} h_{+}^{a_{2}} \ldots h_{+}^{a_{m}} h_{+}^{c}\right)\right] \\
+ & \left.\left[f^{b a_{1} c}\left(b_{0}^{c}-2 i h_{0}^{c}\right) h_{+}^{a_{2}} \ldots h_{+}^{a_{m+1}}\right]\right\}
\end{array}
$$

where we have made repeated use of invariance of $d$ to eliminate certain ultralocal and non-ultralocal terms. The surviving terms written above can now be grouped together into two sets proportional to $\mathbf{d}\left(h_{+}^{m+1}\right)=0$ and $\mathbf{d}\left(j_{+} h_{+}^{m}\right)=0$. Hence the bracket vanishes.

Finally we consider the bracket of $Q^{(1) a}$ with

$$
\int d x d_{a_{1} a_{2} a_{3} \ldots a_{m+1}}\left(m j_{+}^{a_{1}} j_{+}^{a_{2}}+2 i \psi_{+}^{a_{1}} \partial_{1} \psi_{+}^{a_{2}}-i\left(j_{-}^{a_{1}}+\frac{1}{2} i h_{-}^{a_{1}}\right) h_{+}^{a_{2}}\right) h_{+}^{a_{3}} \ldots h_{+}^{a_{m+1}},
$$

which we know differs from $B_{m}^{+}$by a term proportional to $B_{m}^{-}$. The resulting expression has three groups of three [...] terms, one for each of $j^{2}, \psi \partial_{1} \psi$ and $\left(j_{-}+\frac{1}{2} i h_{-}\right) h_{+}$:

$$
\begin{aligned}
\left\{Q^{(1) b}, B_{m}^{+}\right\}= & \int d x d_{a_{1} a_{2} \ldots a_{m+1}}\left\{m\left[\left(f^{b a_{1} c} j_{+}^{a_{2}}+f^{b a_{2} c} j_{+}^{a_{1}}\right) b_{1}^{c} h_{+}^{a_{3}} \ldots h_{+}^{a_{m+1}}\right]\right. \\
& +m\left[-i\left(f^{b a_{1} c} j_{+}^{a_{2}}+f^{b a_{2} c} j_{+}^{a_{1}}\right)\left(2 h_{1}^{c}+h_{0}^{c}\right) h_{+}^{a_{3}} \ldots h_{+}^{a_{m+1}}\right. \\
& \left.\quad+j_{+}^{a_{1}} j_{+}^{a_{2}}\left(f^{b a_{3} c} h_{+}^{c} h_{+}^{a_{4}} \ldots h_{+}^{a_{m+1}}+\ldots+f^{b a_{m+1} c} h_{+}^{a_{3}} \ldots h_{+}^{a_{m}} h_{+}^{c}\right)\right] \\
& +m\left[\left(f^{b a_{1} c} j_{+}^{a_{2}}+f^{b a_{2} c} j_{+}^{a_{1}}\right) j_{0}^{c} h_{+}^{a_{3}} \ldots h_{+}^{a_{m+1}}\right] \\
& +[0]+[0]+\left[-4 i f^{b a_{1} c} j_{0}^{c} h_{+}^{a_{2}} \ldots h_{+}^{a_{m+1}}\right] \\
& +\left[-i f^{b a_{1} c} b_{1}^{c} h_{+}^{a_{2}} \ldots h_{+}^{a_{m+1}}\right] \\
& +\left[-i\left(b_{0}^{a_{1}}-b_{1}^{a_{1}}-2 i h_{0}^{a_{1}}\right)\left(f^{b a_{2} c} h_{+}^{c} h_{+}^{a_{3}} \ldots h_{+}^{a_{m+1}}+\ldots+f^{b a_{m+1} c} h_{+}^{a_{2}} \ldots h_{+}^{a_{m}} h_{+}^{c}\right)\right. \\
& \\
& \left.+\left[i f^{b a_{1} c} j_{0}^{c} h_{+}^{a_{2}} \ldots h_{+}^{a_{m+1}}\right]\right\} .
\end{aligned}
$$


As before, there is some work to be done to show that other terms, besides those written above, vanish along the way. The second [0] in the middle line is due to a total derivative. Gathering together the terms containing the factors $m$ yields, after a little rearranging,

$$
m\left\{\mathbf{d}\left(j_{+}^{2} h_{+}^{m-1}\right)-2 i \underline{h_{+}} j_{+} h_{+}^{m-1}\right\} .
$$

Now we add the rest of the terms, which are

$$
-i \underline{\left(3 j_{0}-2 i h_{1}+b_{1}\right)} h_{+}^{m}-i m\left(b_{0}-b_{1}-2 i h_{0}\right) \underline{h_{+}} h_{+}^{m-1}=-i \mathbf{d}\left(\left(b_{0}-b_{1}-2 i h_{0}\right) h_{+}^{m}\right)-2 i \underline{j_{+}} h_{+}^{m} .
$$

The end result is

$$
-2 i \underline{j_{+}} h_{+}^{m}-2 i m j_{+} \underline{h_{+}} h_{+}^{m-1}=-2 i \mathbf{d}\left(j_{+} h_{+}^{m}\right)=0 .
$$

\section{Appendix: Invariant tensors}

This section is a comprehensive guide to the invariant tensors of relevance to this paper. As above, $\mathcal{G}$ is a Lie group with algebra $\mathbf{g}$ and $\operatorname{rank}(\mathbf{g})=l$. Fundamental representation generators are $\left\{t_{a}\right\}$ and they satisfy

$$
\operatorname{Tr}\left(t_{a} t_{b}\right)=-\delta_{a b}, \quad\left[t_{a}, t_{b}\right]=f_{a b c} t_{c}
$$

An arbitrary tensor $\Theta_{a_{1} \ldots a_{n}}$ is called invariant if we have

$$
\sum_{k=1}^{n} f_{b a_{k}}^{c} \Theta_{a_{1} \ldots a_{k-1} c a_{k+1} \ldots a_{n}}=0
$$

An equivalent statement is that the element of the enveloping algebra of $\mathbf{g}$ given by

$$
\hat{\Theta}=\Theta_{a_{1} a_{2} \ldots a_{n}} t_{a_{1}} t_{a_{2}} \ldots t_{a_{n}}
$$

is a Casimir, that is $\left[t_{b}, \hat{\Theta}\right]=0$ for every $t_{b}$. Symmetrizations and antisymmetrizations are denoted by

$$
\Theta_{\left(a_{1} \ldots a_{n}\right)}=\frac{1}{n !} \sum_{\sigma} \Theta_{a_{\sigma(1)} \ldots a_{\sigma(n)}}, \quad \Theta_{\left[a_{1} \ldots a_{n}\right]}=\frac{1}{n !} \sum_{\sigma}(-1)^{\sigma} \Theta_{a_{\sigma(1)} \ldots a_{\sigma(n)}}
$$

respectively, where the sums extend over all permutations $\sigma$ of $\{1,2, \ldots, n\}$ and $(-1)^{\sigma}$ denotes the signature of the permutation. 


\subsection{Primitive symmetric tensors}

There are $l$ primitive symmetric tensors for each algebra $\mathbf{g}$. What this means is that any symmetric invariant tensor can be expressed as a sum of tensor products of these primitive tensors. The primitive tensors are not unique, but the ambiguity in their selection consists of the freedom to add or subtract symmetrized tensor products, of the form

$$
d_{a_{1} \ldots a_{n}}=u_{\left(a_{1} \ldots a_{k}\right.} v_{\left.a_{k+1} \ldots a_{n}\right)}
$$

(up to overall constants). In section 2 we introduced the term 'compound' for such tensors. For the classical algebras, we can take all but one of the primitive tensors to be

$$
s_{a_{1} a_{2} \ldots a_{n}}=\sin \left(t_{a_{1}} t_{a_{2}} \ldots t_{a_{n}}\right)=\operatorname{Tr}\left(t_{\left(a_{1}\right.} t_{a_{2}} \ldots t_{\left.a_{n}\right)}\right)
$$

where $n$ takes the values

$$
\begin{aligned}
a_{l}=s u(l+1) & 2,3, \ldots(l+1) \\
b_{l}=s o(2 l+1) & 2,4, \ldots 2 l \\
c_{l}=s p(2 l) & 2,4, \ldots 2 l \\
d_{l}=s o(2 l) & 2,4, \ldots(2 l-2)
\end{aligned}
$$

Note that this only defines $(l-1)$ tensors for the algebras $d_{l}$. The final invariant in this case is the Pfaffian, given by

$$
p_{a_{1} \ldots a_{l}}=\frac{1}{2^{l} l !} \epsilon^{j_{1} \ldots j_{2 l}}\left(t_{a_{1}}\right)_{j_{1} j_{2}} \ldots\left(t_{a_{l}}\right)_{j_{2 l-1} j_{2 l}} .
$$

To illustrate these ideas, consider the algebra $a_{3}$. This algebra has rank three and a set of primitive symmetric tensors is provided by

$$
\operatorname{Tr}\left(t_{a_{1}} t_{a_{2}}\right), \quad \mathrm{s} \operatorname{Tr}\left(t_{a_{1}} t_{a_{2}} t_{a_{3}}\right), \quad \mathrm{s} \operatorname{Tr}\left(t_{a_{1}} t_{a_{2}} t_{a_{3}} t_{a_{4}}\right)
$$

The six-fold symmetric trace is non-primitive and can be written

$$
\begin{aligned}
\mathrm{s} \operatorname{Tr}\left(t_{a_{1}} t_{a_{2}} t_{a_{3}} t_{a_{4}} t_{a_{5}} t_{a_{6}}\right) & =\frac{1}{3} \operatorname{Tr}\left(t_{\left(a_{1}\right.} t_{a_{2}} t_{a_{3}}\right) \operatorname{Tr}\left(t_{a_{4}} t_{a_{5}} t_{\left.a_{6}\right)}\right) \\
& +\frac{3}{4} \operatorname{Tr}\left(t_{\left(a_{1}\right.} t_{a_{2}}\right) \operatorname{Tr}\left(t_{a_{3}} t_{a_{4}} t_{a_{5}} t_{\left.a_{6}\right)}\right) \\
& -\frac{1}{8} \operatorname{Tr}\left(t_{\left(a_{1}\right.} t_{a_{2}}\right) \operatorname{Tr}\left(t_{a_{3}} t_{a_{4}}\right) \operatorname{Tr}\left(t_{a_{5}} t_{\left.a_{6}\right)}\right) .
\end{aligned}
$$




\section{$9.2 \Omega$ tensors}

Given any symmetric invariant tensor $d_{a_{1} \ldots a_{n}}^{(n)}$ we can define an order $(2 n-1)$ antisymmetric invariant tensor by

$$
\begin{aligned}
\Omega_{a_{1} a_{2} \ldots a_{2 n-1}}^{(2 n-1)} & =\frac{1}{2^{n-1}} f_{\left[a_{1} a_{2}\right.}^{b_{1}} f_{a_{3} a_{4}}^{b_{2}} \ldots f_{a_{2 n-3} a_{2 n-2}}^{b_{n-1}} d^{b_{1} b_{2} \ldots b_{n-1}}{ }_{\left.a_{2 n-1}\right]} \\
& =\frac{1}{2^{n-1}} f_{a_{1}\left[a_{2}\right.}^{b_{1}} f_{a_{3} a_{4}}^{b_{2}} \ldots f_{\left.a_{2 n-3} a_{2 n-2}\right]}^{b_{n-1}} d^{b_{1} b_{2} \ldots b_{n-1}}{ }_{a_{2 n-1}}
\end{aligned}
$$

The second equality follows from careful use of invariance properties [9]. We also observe that for any symmetric invariant tensor $d$,

$$
d_{a_{1} \ldots a_{n}} f_{\left[b_{1} b_{2}\right.}^{a_{1}} \ldots f_{\left.b_{2 n-1} b_{2 n}\right]}^{a_{n}}=0
$$

by using invariance and the Jacobi identity. This leads to a very useful property of the $\Omega$ tensors: if $d$ in (9.8) is compound, as in (9.5), then $\Omega$ vanishes identically. This is easily understood by considering the symmetrization of indices in (9.5) to be written out explicitly, followed by substitution in (9.8). In every one of the resulting terms either $u$ or $v$ has all its indices contracted with structure constants, as in (9.9), and the result follows. Since (9.8) is linear in $d$, the expression for $\Omega$ will also vanish if $d$ is any sum of compound tensors. The corollary to this is that only the primitive part of $d$ contributes to $\Omega$. From the primitive symmetric tensors (9.6) we obtain

$$
\Omega_{a_{1} \ldots a_{2 n-1}}=\operatorname{Tr}\left(t_{\left[a_{1}\right.} t_{a_{2}} \ldots t_{\left.a_{2 n-1}\right]}\right)
$$

providing $l$ primitive antisymmetric tensors for $a_{l}, b_{l}$ and $c_{l}$. For $d_{l}$, we have $l-1$ tensors of this form and the final primitive antisymmetric tensor is that defined from the Pfaffian via (9.8). In general, we have precisely $l$ primitive totally antisymmetric invariant tensors, which are in 1-1 correspondence with the primitive symmetric tensors of $\mathbf{g}$.

\section{3 $\Lambda$ tensors}

Given a general symmetric invariant tensor $d_{a_{1} a_{2} \ldots a_{n}}^{(n)}$ we define a $\Lambda$ tensor by

$$
\Lambda_{a_{1} a_{2} \ldots a_{2 n-2}}^{(2 n-2)}=\frac{1}{2^{n-2}} f_{\left[a_{1} a_{2}\right.}^{b_{1}} \ldots f_{a_{2 n-5} a_{2 n-4}}^{b_{n-2}} d^{b_{1} b_{2} \ldots b_{n-2}}{ }_{\left.a_{2 n-3}\right] a_{2 n-2}}
$$

As these tensors have less symmetry than the $\Omega$ tensors we would expect there to be a larger class of them. In the above section, we saw that only the primitive part of the 
$d$-tensor contributed to $\Omega$. We would like to know what is the analogue of this for the $\Lambda$ tensors. The answer is that compound tensors (9.5) may contribute to $\Lambda$, but not if they can be written as a product of three of more factors:

$$
d_{a_{1} \ldots a_{n}}=u_{\left(a_{1} \ldots a_{r}\right.} v_{a_{r+1} \ldots a_{s}} w_{\left.a_{s+1} \ldots a_{n}\right)}
$$

As before, we need only think of such a compound tensor used in the definition of $\Lambda$ above, with the symmetrization on its indices written out. In each of the resulting terms, at least one of the constituents $u, v$ or $w$ will have all its indices contracted with structure constants, and so will vanish by (9.9).

It remains to understand how compound tensors involving just two primitive constituents contribute to $\Lambda$. This involves nothing more than substituting a general tensor of this type

$$
d_{a_{1} \ldots a_{p} b_{1} \ldots b_{q}}=d_{\left(a_{1} \ldots a_{p}\right.}^{(p)} d_{\left.b_{1} \ldots b_{q}\right)}^{(q)}
$$

into the definition. Some care is required with combinatorial factors, however, in order to arrive at the result

$$
\Lambda_{a_{1} \ldots a_{2 n-1} b}^{(2 n)}=\frac{p q}{(p+q)(p+q-1)}\left(\Omega_{\left[a_{1} \ldots a_{2 p-1}\right.}^{(2 p-1)} \Omega_{a_{2 p} \ldots a_{2 n-1} b}^{(2 q-1)}+\Omega_{\left[a_{1} \ldots a_{2 q-1}\right.}^{(2 q-1)} \Omega_{a_{2 q} \ldots a_{2 n-1} b}^{(2 p-1)}\right)
$$

where $n=p+q-1$ and $\Omega^{(2 p-1)}$ and $\Omega^{(2 q-1)}$ are related to $d^{(p)}$ and $d^{(q)}$ as in (9.8). Unlike the $\Omega$ tensors, there is not a unique $\Lambda$ tensor for each primitive symmetric invariant. Nevertheless, we see that $\Lambda$ tensors based on different $d$ tensors will differ only by linear combinations of products of $\Omega$ tensors, as in the expression above.

\subsection{Comments}

There are various awkward coefficients which arise in checking some statements made in section 4.2 relating to the bosonic terms in the definition $(4.11)$ of the currents $\mathcal{K}_{m+1}^{+}$for $s u(N)$. It was found that commuting charges could be obtained by modifying the current $\mathcal{B}_{m+1}^{+}$by an expression including

$$
-\frac{m}{N} \sum_{p+q=m+1} \mathcal{B}_{p}^{-} \mathcal{B}_{q}^{-}
$$

Considering first how these quantities can be written in terms of symmetric tensors, the modification amounts to changing $s_{a_{1} a_{2} a_{3} \ldots a_{m+1}}^{(m+1)} j_{+}^{a_{1}} j_{+}^{a_{2}} h_{+}^{a_{3}} \ldots h_{+}^{a_{m+1}}$ by

$$
-\frac{1}{N} \sum_{p+q=m+1}\left(s_{a_{1} a_{2} \ldots a_{p}}^{(p)} j_{+}^{a_{1}} h_{+}^{a_{2}} \ldots h_{+}^{a_{p}}\right)\left(s_{b_{1} b_{2} \ldots b_{q}}^{(q)} j_{+}^{b_{1}} h_{+}^{b_{2}} \ldots h_{+}^{b_{q}}\right)
$$




$$
=-\frac{1}{N} \sum_{p+q=m+1} \frac{(p+q)(p+q-1)}{2 p q} s_{\left(a_{1} a_{2} \ldots a_{p}\right.}^{(p)} s_{\left.b_{1} b_{2} \ldots b_{q}\right)}^{(q)} j_{+}^{a_{1}} j_{+}^{b_{1}} h_{+}^{a_{2}} \ldots h_{+}^{a_{p}} h_{+}^{b_{2}} \ldots h_{+}^{b_{q}}
$$

where care must be taken with symmetrizations in order to obtain the correct coefficents in the second expression. This is easily found to reproduce the compound terms with two primitive factors which appear in the tensors $k^{(m+1)}$ listed in (2.38) in section 2. Alterna-

tively, in terms of antisymmetric tensors, we need to modify $\Lambda_{a_{1} a_{2} \ldots a_{2 m-1} b}^{(2 m)} j_{+}^{a_{1}} \psi_{+}^{a_{2}} \ldots \psi_{+}^{a_{2 m-1}} j_{+}^{b}$ by the expression

$$
-\frac{1}{N} \frac{m}{2 m-1} \sum_{p+q=m+1}\left(\Omega_{a_{1} a_{2} \ldots a_{2 p-1}}^{(2 p-1)} j_{+}^{a_{1}} \psi_{+}^{a_{2}} \ldots \psi_{+}^{a_{2 p-1}}\right)\left(\Omega_{b_{1} b_{2} \ldots b_{2 q-1}}^{(2 q-1)} j_{+}^{b_{1}} \psi_{+}^{b_{2}} \ldots \psi_{+}^{b_{2 q-1}}\right) .
$$

Now it can be checked that

$$
\begin{aligned}
& \left(\Omega_{\left[a_{1} a_{2} \ldots a_{2 p-1}\right.}^{(2 p-1)} \Omega_{\left.a_{2 p} \ldots a_{2 m-1}\right] b}^{(2 q-1)}+\Omega_{\left[a_{1} a_{2} \ldots a_{2 q-1}\right.}^{(2 q-1)} \Omega_{a_{2 q} \ldots a_{2 m-1} b}^{(2 p-1)}\right) j_{+}^{a_{1}} \psi_{+}^{a_{2}} \ldots \psi_{+}^{a_{2 m-1}} j_{+}^{b} \\
= & \frac{2 p+2 q-2}{2 p+2 q-3}\left(\Omega_{a_{1} a_{2} \ldots a_{2 p-1}}^{(2 p-1)} j_{+}^{a_{1}} \psi_{+}^{a_{2}} \ldots \psi_{+}^{a_{2 p-1}}\right)\left(\Omega_{b_{1} b_{2} \ldots b_{2 q-1}}^{(2 q-1)} j_{+}^{b_{1}} \psi_{+}^{b_{2}} \ldots \psi_{+}^{b_{2 q-1}}\right)
\end{aligned}
$$

from which we see that (9.17) is equal to

$$
-\frac{1}{N} \sum_{p=2}^{m-1} \Omega_{\left[a_{1} \ldots a_{2 r-1}\right.}^{(2 p-1)} \Omega_{\left.a_{2 p} \ldots a_{2 m-1}\right] b}^{(2 m-2 p+1)} j_{+}^{a_{1}} \psi_{+}^{a_{2}} \ldots \psi_{+}^{a_{2 m-1}} j_{+}^{b}
$$

precisely as required for (4.12). Notice also that this is consistent with (9.16) above on using (9.14).

\section{References}

[1] J.M. Evans, M. Hassan, N.J. MacKay, and A.J. Mountain, Local conserved charges in principal chiral models, Nucl. Phys. B561 (1999) 385; hep-th/9902008.

[2] J.M. Evans, M. Hassan, N.J. MacKay, and A.J. Mountain, Conserved charges and supersymmetry in principal chiral models, based on conference talks by JME, NJM and AJM; hep-th/9711140v3 (January 1999).

[3] E. Corrigan, Recent developments in affine Toda quantum field theory, Lectures given at CRM-CAP Summer School on Particles and Fields '94, Banff, Canada, 16-24 Aug 1994, preprint DTP-94/55; hep-th/9412213.

[4] P.E. Dorey, Root systems and purely elastic S-matrices, Nucl. Phys. B358 (1991) 654 
[5] V. Chari and A. Pressley, Yangians, integrable quantum systems and Dorey's rule, Commun. Math. Phys. 181 (1996) 265; hep-th/9505085.

[6] E. Ogievetsky, N. Reshetikhin and P. Wiegmann, The principal chiral field in two dimensions on classical Lie algebras: the Bethe ansatz solution and factorized theory of scattering, Nucl. Phys. B280 (1987) 45.

[7] E. Witten, Non-Abelian bosonization in two dimensions, Commun. Math. Phys. 92 (1984) 455.

[8] J. Balog, L. Fehér, L. O’Raifeartaigh, P. Forgács, A. Wipf, Toda theory and W-algebra from a gauged WZNW point of view, Ann. Phys. 203 (1990) 76.

[9] J.A. de Azcárraga, A.J. Macfarlane, A.J. Mountain and J.C. Pérez Bueno, Invariant tensors for simple groups, Nucl. Phys. B510 (1998) 657; physics/9706006.

[10] A.J. Mountain, Invariant tensors and Casimir operators for simple compact Lie groups, J. Math Phys. 39 (1998) 5601; physics/9802012.

[11] N.J. MacKay, On the classical origins of Yangian symmetry in integrable field theory, Phys. Lett. B281 (1992), 90; err. ibid. B308 (1993) 444.

[12] E. Brézin, C. Itzykson, J. Zinn-Justin and J.-B. Zuber, Remarks on the existence of non-local charges in two-dimensional models, Phys. Lett. 82B (1979) 442.

[13] D. Bernard, On symmetries of some massless 2D field theories, Phys. Lett. B279 (1992) 78.

[14] S. Ferrara, L. Giradello and S. Sciuto, An infinite set of conservation laws in the supersymmetric sine-Gordon theory, Phys. Lett. B76 (1978) 303.

[15] P.S. Howe and G. Papadopoulos, Holonomy groups and W-symmetries, Commun. Math. Phys. 151 (1993) 467; hep-th/9202036.

[16] E. Abdalla, M.C.B. Abdalla, J.C. Brunelli and A. Zadra, The algebra of non-local charges in non-linear sigma models, Commun. Math. Phys. 166 (1994) 166.

M.C.B. Abdalla, Integrability of chiral nonlinear sigma models with a Wess-Zumino term, Phys. Lett. B152 (1985) 215.

[17] H. J. de Vega, Field theories with an infinite number of conservation laws and Bäcklund transformations in two dimensions, Phys. Lett. B87 (1979) 233. 
[18] A.V. Mikhailov, Integrability of supersymmetric generalization of classical chiral models in two-dimensional space-time, JETP Lett. 28 (1978) 512.

Z. Popowicz and L.-L. Chau Wang, Backlund transformation, local and non-local conservation laws for super-chiral fields, Phys. Lett. B98 (1981) 253.

E. Abdalla and M. Forger, Integrable non-linear $\sigma$-models with fermions, Commun. Math. Phys. 104 (1986) 123.

[19] J.M. Evans and T.J. Hollowood, Exact scattering in the SU(N) supersymmetric principal chiral model, Nucl. Phys. B493 (1997) 517; hep-th/9603190.

[20] P. Di Vecchia, V.G. Knizhnik, J.L. Petersen and P. Rossi, A supersymmetric Wess-Zumino lagrangian in two dimensions, Nucl. Phys. B253 (1985) 701.

[21] J. Fuchs, Superconformal Ward identities and the WZW model, Nucl. Phys. B286 (1987) 455; More on the super WZW theory, Nucl. Phys. B318 (1989) 631.

[22] Y.Y. Goldschmidt and E. Witten, Conservation laws in some two-dimensional models, Phys. Lett. B91 (1980) 392.

[23] T. E. Clark, S. T. Love and S. Gottlieb, Infinite number of conservation laws in two-dimensional superconformal models, Nucl. Phys. B186 (1981) 347.

[24] T. Curtright and C. Zachos, Nonlocal currents for supersymmetric nonlinear models, Phys. Rev. D21 (1980) 411; Supersymmetry and the nonlocal Yangian deformation symmetry, Nucl. Phys. B402 (1993) 604.

E. Corrigan and C. Zachos, Non-local charges for the supersymmetric $\sigma$-model, Phys. Lett. B88 (1979) 273.

[25] L.-L. Chau and H. C. Yen, Integrability of the super-chiral model with a Wess-Zumino term, Phys. Lett. B177 (1986) 368.

[26] G. Papadopoulos, Supersymmetric Toda field theories, Phys. Lett. B365 (1996) 98; hep-th/9508175.

[27] J.M. Evans and J.O. Madsen, Integrability vs Supersymmetry, Phys. Lett. B389 (1996) 665; hep-th/9608190. 